\title{
Ueber \\ die Erregbarkeit der motorischen Nerven an verschiedenen Stellen ihres Verlaufes. \\ Von
}

stud. med. K. Eickhof aus Meschede.

(Mit 5 Textfiguren und Tafel I.)

Wie Jedem bekannt, besitzen alle Nerven die merkwürdige Eigenschaft, durch geeignete Einwirkungen, welche sie in ihrem Verlauf treffen, in Erregung versetzt zu werden, obwohl im normalen Leben des Nerven im Organismus derartige in ihren Verlauf gesetzte Erregungen ebensowenig vorkommen, wie ein Zerren oder Reissen an der Mitte einer Klingelschnur. So wie hier regelrecht nur an dem Ende der Klingelschnur gezogen wird, so wird auch jedweder Nerv unter normalen Bedingungen nur von seinem Endorgan aus erregt und nicht in der Mitte oder an irgend einem Punkte seines Verlaufes.

Ohne diese, ich möchte fast sagen, liebenswürdige Eigenschaft des Nerven, auch in seinem Verlauf erregt zu werden, würde es eine Nervenphysiologie eigentlich kaun geben.

Daher ist es begreiflich, dass die Physiologen dieser Eigenschaft von jeher ihre Aufmerksamkeit zugewendet und namentlich des Genaueren untersucht haben, ob die gleichen Einwirkungen an verschiedenen Stellen des Nerven auch die gleichen Erfolge in dem Endorgan - gewöhnlich dem Muskel - auslösten oder ob eine verschiedene "Erregbarkeit" an verschiedenen Stellen seines Verlaufes bestand, d. h. ob ein und dieselben Einwirkungen oder Reize, je nach dem Orte, an welchem man sie anbrachte, verschiedene Erfolge bedingten.

Indem jeh aus der grossen Anzahl der hierüber angestellten Arbeiten von älteren nur diejenigen von $\mathrm{Budge}{ }^{1}$ ), Pflüger ${ }^{2}$ ).

1) J. Budge, Frorieps Tageberichte Nr. 445 S. 329 und Nr. 905 S. 348 , und Virchow's Archiv Bd. 18 S. 457. 1860.

2) E. Pflüger, Unters. über die Physiol. des Elektrotonus S. 140. 1859. 
und Heidenhain ${ }^{1}$ ) erwähne, welche an dem hoch durchschnittenen Hüftnerven des Frosches eine grössere Erregbarkeit an seinen oberen Abschnitten feststellen konnten (die $\mathrm{Heidenhain} \mathrm{lediglich} \mathrm{als} \mathrm{eine}$ Wirkung des Quersehnittes ansah), gibt es auch eine grössere Anzahl von namentlich neueren Forschern, welche dem völlig unverletzten Nerv in der ganzen Länge seines Verlaufes die gleiche Erregbarkeit zuschreiben. Und schliesslich fehlt auch eine der ersten Angabe nahezu entgegengesetzte nicht, dass nämlich die Erregbarkeit des Nerven in den peripheren Abschnitten seines Verlaufes eine grössere sei als in den centralen.

Was zunächst die elektrische Reizung des Froschischiadicus anlangt, so ergab sich weiter, dass in den oberen Abschnitten absteigende und in den unteren aufsteigende Ströme wirksamer waren. Es konnte ferner gezeigt werden, dass diese merkwürdige Eigenthümlichkeit von Bestandströmen im Nerven herrührte, mit denen entweder die Reizströme gleichgerichtet und dann besonders wirksam, oder denen sie entgegengesetzt gerichtet und dann weniger wirksam waren. Erzeugte man, wie dies Grützne ${ }^{2}$ ) that, künstlich im Nerven durch bestimmte Eingriffe besondere Bestandströme, so komten die obigen von Hermann und von v. Fleischl festgestellten Thatsachen noch erweitert werden. Somit ergab sich die verschiedene elektrische Erregbarbeit des Hüftnerven vom Frosch als eine mehr zufällige, durch die Präparation, beziehungsweise durch das Abschneiden rer Oberschenkeläste bedingte.

Neuerdings baben nun ziemlich gleichzeitig $\mathrm{O} . \mathrm{We}$ iss ${ }^{3}$ ), sowie J. Munk und P. Schultz ${ }^{4}$ ) an dem Hüftnerven des Frosches, wie an langen, ungetheilt verlaufenden Nerven des Säugethiers (Phrenicus, Vagus) nachgewiesen, dass dieselben elektrischen Reizen gegenüber (und zwar constanten Strömen und Oeffnungsinductionsschlägen) nahezu die gleiche Erregbarkeit darbieten. Nur Schädioungen der Nerven an ihrem centralen Ende oder in ihrem Verlauf bedingten verschiedene örtliche Erregbarkeit.

Dieselbe Behauptung, dass der Nerv durchweg die gleiche Er-

1) R. Heid enhain, Studien des physiol. Instituts zu Breslau Heft I S. 1. 1866.

2) P. Grützner, Pflüger's Archiv Bd. 28 S. 130. 1882.

3) O. Weiss, Pflüger's Archiv Bd. 72 S. 15. 1898, woselbst auch die gesammte unser Thema angehende Literatur sorgfältig. zusammengestellt ist, und eluenda Bd. 75 S. 265. 1899.

4) J. Munk und P. Schultz, Archiv für (Anat. und) Physiol. 1898. S. 297. 
regbarkeit habe, war für mechanisehe Reize von Tigerstedt') für den Ischiadicus des Frosches ausgesprochen und die Thatsache der überall gleichen Erregbarkeit als allgemein gültig von verschiedenen Forschern angenommen worden.

Nur Beck ${ }^{2}$ ), worauf hier noch hingewiesen sei, findet an dem Phrenicus und Sympathicus von Warmblütern in den unteren, peripheren Abschnitten gegenüber elektrischen Reizen (Inductionsströmen und Condensatorentladungen) eine grössere Erregbarkeit als in den höheren, centralen Abschnitten, was wie gesagt, von Weiss bestritten wird.

Dieser dagegen erwähnt in seinen beiden Abhandlungen, dass der Beckentheil des Nervus ischiadicus doch eine gewisse Sonderstellung einnimmt, indem seine Erregbarkeit sich als etwas grösser, als die des eigentlichen Nervenstammes im Oberschenkel erwies, und dass er niemals gleich erregbar in seinem Verlauf war. Freilich war er auch hier nie stromlos ${ }^{3}$ ).

Dass num in der That die oberen Abschnitte dieses Nerven, wenı sie vielleicht auch bei schonendster Präparation in dem sonst unverletzten Thiere nahezu dieselbe elektrische Erregbarkeit darbieten, wie die unteren, peripheren, doch diesen weder anatomisch noch physiologisch vollkommen gleich zu stellen sind, wie etwa zwei Stellen eines und desselben Drahtes, das ging aus verschiedenen Untersuchungen von Grützner ${ }^{4}$ ) and seinen Schülern Halperson ${ }^{5}$ ), Efron ${ }^{6}$ ), Groves ${ }^{7}$ ) hervor, welche unzweifelhaft ergaben, dass eben jene oberen Abschnitte gegenüber verschiedenen anderen Eingriffen empfindlicher sind, dass sie vielleicht desshalb zwar meistens besser erregbar, daneben aber auch viel leichter verletzbar sind ${ }^{8}$ ).

1) R. Tigerstedt, Studien über mechan. Nervenreizung. Helsingfors 1880 S. 61 .

2) A. Beck, Archiv für (Anat. und) Physiol. 1897. S. 415 und Pflüger's Archiv Bd. 72 S. 352.1898.

3) 0. Weiss, Pflüger's Archiv Bd. 72 S. 22 und Bd. 75 S. 276.

4) P. Grützner, Pflüger's Archiv Bd. 53 S. 83. 1892.

5) Clara Halperson, Elektr. Erregbarkeit der Nervenfasern. Dissert. Bern 1884 .

6) J. Efron, Pflüger's Archiv Bd. 36 S. 467. 1885.

7) E. W. Groves, Journal of physiology vol. 14 p. 221. 1893.

8) Uebrigens sei hier daranf hingewiesen, dass auch Helmholtz mit Baxt (Berliner Akademieber. 1867, Berlin 1868 S. 228 (231) in dem Arm des Menschen den Nervus medianus gegenüber elektrischen Reizen oben viel erregbarer fanden, als üten. Auch Place (Pflüger's Arch. Bd. 3. 1870. S. 430) macht dieselbe Angabe. 
Von diesem Gesichtspunkte aus unternahm ich es nun, verschiedene Reize auf die oberen und unteren Stellen des Froschischiadicus zu untersuchen, und da es keinen nennenswerthen Unterschied machte, ob der Nerv hoch oben durchschnitten war oder mit dem Rückenmarkstumpf in Verbindung blieb, so habe ich meist an zerschnittenen Hüftnerven experimentiert und habe zunächst einige unter sich verschiedene elektrische Reize geprüft, die, wie mir Herr Prof. Grütz$\mathrm{n}$ er mittheilte, ihm bei gelegentlichen Beobachtungen eigenthümliche Verschiedenheiten in ihren Wirkungen ergeben hatten.

Diese Untersuchungen stellte ich im Sommerhalbjahr 1898 in dem Tübinger physiologischen Institut an und hatte mich dabei der andauernden Leitung und Unterstützung von Seiten meines hochgeschätzten Lehrers zu erfreuen. Mit besonderer Freude spreche ich ihm sowohl hierfür, wie für die mir bei der Abfassung der folgenden Arbeit geleistete Hilfe, meinen besten Dank aus. Auch Herr Dr. Fuld widmete mir vielfach Zeit und Mühe, so dass ich auch ihm zu Dank verpflichtet bin.

\section{Eigene Untersuchungen.}

1. Die örtlich verschiedene Erregbarkeit des Hüftnerven gegenüber schnell und langsam verlaufenden elektrischen Strömen.

Da nach den oben erwähnten Untersuchungen an dem Hüftnerven des Frosches, namentlich an seinem Beckentheil, andere Erregbarkeitsverhältnisse nacbgewiesen werden konnten, als an seinen unteren Stellen nahe am Kniee, und da weiter die oberen Abschnitte sich als empfindlichere und beweglichere Elemente erwiesen, als die unteren, so lag es nahe, elektrische Reizströme von verschiedenem Verlauf anzuwenden. Den trägen Nerven beziehungsweise trägen Endapparaten gegenüber erwiesen sich langsam verlaufende Reizströme oft wirksamer, als schnell verlaufende, während flinken Nerven, die auch mit flinken Endapparaten in Verbindung stehen, schnell und jäh verlaufende Reize besser zusagten.

Obwohl diese von Grützner und Schotti) festgestellten Thatsachen keineswegs allgemeine Geltung beanspruchen, sondern,

1) J. Schott, Pflüger's Archiv Bd. 48 S. 354.1891. 
wie Bürker ${ }^{1}$ ) zeigte, auch Nerven, welche mit trägen Apparaten, wie Drüsen, trägen (rothen) Muskeln in Verbindung stehen, besser durch schnell verlaufende Ströme erregt werden, so war es doch anzunehmen, dass diese verschiedenen Ströme an den oberen und unteren Abschnitten des Nerven verschiedene Wirkungen entfalten würden.

Ich verwendete als Reizströme die Ströme eines gewöhnlichen Inductionsapparates, welche aber nach einer von Herrn Prof. Grützner angegebenen, gleich näher zu beschreibenden Art in ihrem Verlauf verändert wurden.

Zunächst galt es nun die Stärke derselben festzustellen, damit man sie miteinander vergleichen konnte. Der von mir angewendete Inductionsapparat wurde also nach der zuerst von $\mathrm{A}$. Fick ${ }^{2}$ ) augegebenen Methode, nebenher auch nach derjenigen von v. Fleischl ${ }^{3}$ ) graduirt. Noch bequemer fand ich es, nicht bloss den Ausschlag der Bussole durch einen einzigen Inductionsschlag zu messen, sondern eine Reihe von gleich viel und zeitlich gleich abständigen Oeffnungsinductionsschlägen bei abgeblendeten Schliessungsinductionsschlägen oder umgekehrt durch die Bussole zu senden.

Um den Verlauf der Schliessungsinductionsschläge möglichst zu verlangsamen, bediente ich mich im Wesentlichen der Methode von J. Henry, welche E. du Bois-Reymond ${ }^{4}$ ) in seiner bekannten Arbeit "Ueber den zeitlichen Verlauf volta-elektrischer Inductionsströme" erwähnt. Henry hatte als Grund der uberaus kräftigen (physiologischen) Wirkung des Oeffnungsinductionsschlages gegenüber dem Schliessungsinductionsschlag wohl als Erster die Thatsache erkannt, „dass sich beim Schliessen in der Hauptrolle der Extrastrom in entgegengesetzter Richtung des Kettenstromes bildet und das Entstehen dieses letzteren verzögert, während beim Oeffnen dem entsprechenden Extrastrom der Weg abgeschnitten ist.

Der Unterschied zwischen denı Schliessungs- und dem Oeffnungs-

1) K. Bürker, Ueber die Errzeugung und physiolog. Wirkung schnell und langsam verlaufender elektrischer Ströme, Dissertation der naturw. Facultät. Tübingen 1897.

2) Untersuchungen aus dem physiolog. Laborat. der Züricher Hochschule. Wien 1869.

3) O. v. Fleischl, Wiener Akademieberichte Abth. 3. 1875.

4) E. du Bois-Reymond, Berliner Akademiebericht 1862 S. 372 und gesammelte Abhand. Bd. 1 S. 228. 1875. 
schlage wird daher um so kleiner, aus je weniger Windungen und je lockerer die Hauptrolle gewickelt ist, da mit der Zahl und Nähe ibrer Windungen die elektromotorische Kraft des Extrastromes wächst." Mit einem Worte, Alles, was das Entstehen und Abgleichen des Extracurrenten fördert, schwächt in hohem Maasse die physiologische Wirkung des Schliessungsinductionsstromes in der secundären Spule, da jener seinen Verlauf verzögert. Wenn man daher ferner auch den Widerstand des primären Kreises möglichst herabsetzt, indem man statt der gewöhnlichen Elemente eine Thermosäule mit ihrem verschwindenden Widerstande anwendet, so kann sich der Extracurrent vortrefflich entwickeln und abgleichen, und den Schliessungsinductionsstrom gegenüber dem Froschnerven beinahe unwirksam machen.

Vergleicht man beide Ströme mit einander im Telephon, so ist der Schliessungsschlag viel schwächer als sonst, ganz leise und matt, ja oft kaum hörbar. Der Oeffunngsschlag dagegen gibt nach wie vor seinen bekannten, lauten, scharfen Knack.

Wenn man nun andererseits das Entstehen und Abgleichen des Extracurrenten erschwert, indem man in den primären Kreis einen grossen Widerstand einschaltet und natürlich durch Vermehrung der Elemente die Stromstärke auf derselben Höhe erhält, so wird der Schliessungsinductionsschlag dem Oeffnungsinductionssehlag in seinem Verlauf und in seiner physiologischen Wirkung genähert, ja er kann ihn sogar noch übertreffen, wie Hen ry erwähnt.

Im Telephon geben dann beide Ströme laute, kurze Knacke, die man kaum von einander unterscheiden kann. Und es ist natürlich nicht schwer, dem Schliessungsschlag sogar das Uebergewicht zu geben.

Da es uns nun wesentlich darauf ankam, langsam und schnell verlaufende Inductionsströme in schnellem Wechsel zu erzeugen und in ihrer physiologischen Wirkung mit einander zu vergleichen, so bedienten wir uns folgender Einrichtung; die nebenstehend schematisch abgebildet ist (s. Fig. 1).

$T$ bedeutet die Noë'sche Thermosäule, welche von einer gut regulirten Spirituslampe erwärmt wurde, $B$ eine Batterie aus mehreren, gewöhnlich 6 kleinen Daniells. Die Elektroden von $T$ und $B$ führen zu einer Wippe ohne Kreuz $W_{1}$. Von hier gehen sie, die eine durch einen Quecksilberschlüssel $S$ unterbrochen, zu einer Wippe mit Kreuz $W_{2}$, die andere durch ein Ampèremeter $A$ ebendahin und schliesslich beide in die primäre Spule I. II stellt die secundäre Spule 
mit den (nicht gezeichneten) unpolarisirbaren Elektroden dar, welche dem Nerven angelegt wurden.

In dem Batteriestromkreis befand sich noch ein Widerstandskasten $R$ von Siemens, in dem Kreis der primären Spule, wie schon gesagt, das Amperemeter. Die Stromstärke schwankte meistens zwischen 28-30 Milliampères. Theils wurde der ganze Unterschenkel an dem sorgfältig präparirten Nerven belassen, und es wurden die zuerst auftretenden schwachen Zuckungen beobachtet, theils zeichnete der Wadenmuskel allein seine Zusammenziehungen auf einem berussten Cylinder auf.

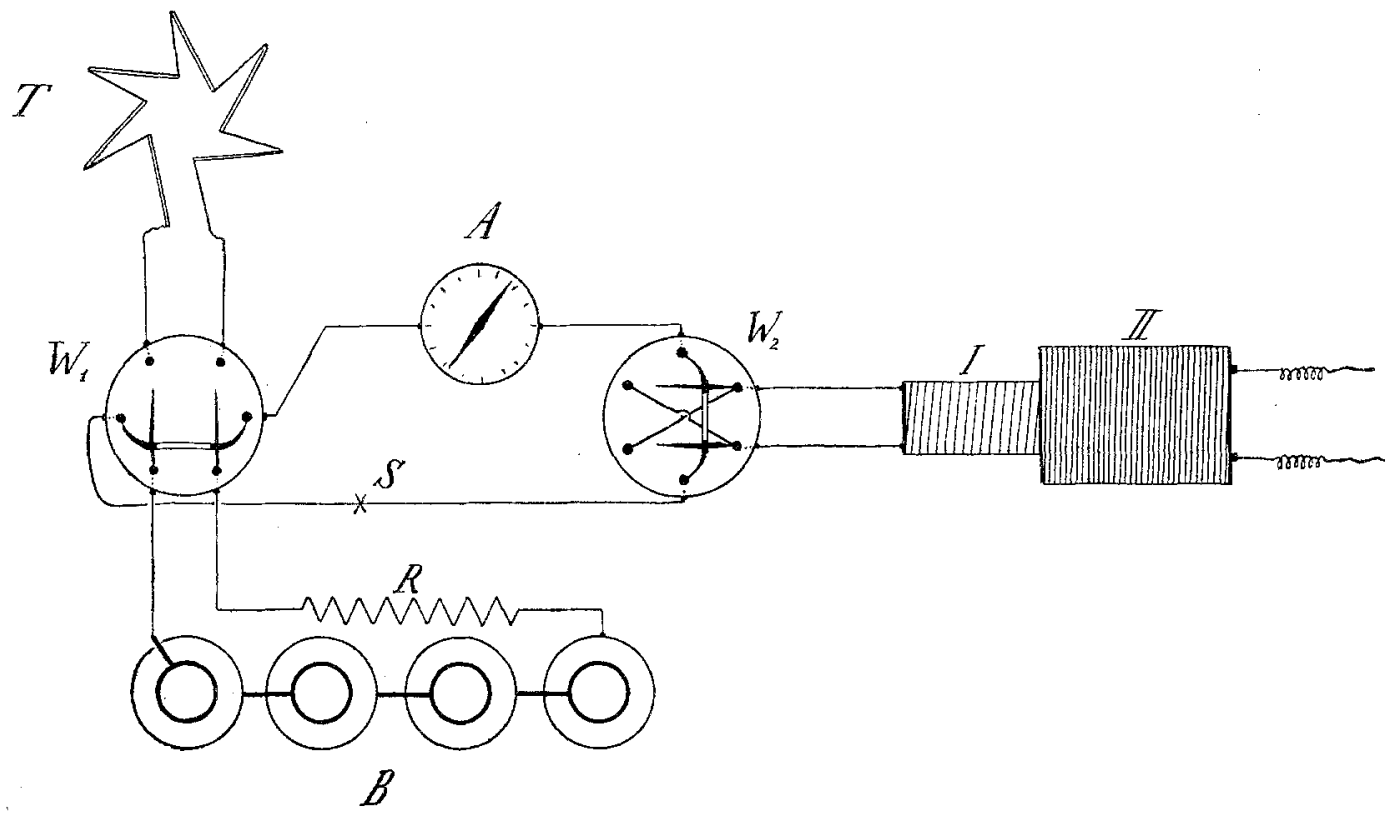

Fig. 1.

Da bekanntlich in den oberen Abschnitten des Nerven, im Plexus, absteigende, in den untern, nahe dem Knie gelegenen, aufsteigende Ströme besser wirksam sind, so mussten wir selbstverständlich zunächst die Ströme günstiger Richtung mit einander vergleichen. Indem wir nun die schnellen Oeffnungsschläge miteinander, sowie die langsamen Schliessungsschläge miteinander in ihren Wirkungen auf die oberen und unteren Theile des Nerven verglichen, erhielten wir ausuahmslos folgende bemerkenswerthe Ergebnisse.

Während die jähen Oeffnungsschläge die geringsten Zuckungen bei nahezu gleicher Stromstärke oben und 
unten erzeugen, muss man die langsam verlaufenden Schliessungschläge (die natürlich an und für sich nach dem hier geltenden Gesetz von Du Bois-Reymond viel stärker sein müssen), auf das Doppelte bis Dreifache ihrer Stärke erhöhen, damit sie unten die gleiche Reizwirkung ausüben wie oben.

Folgende, tabellarisch zusammengestellte Versuche erläutern die Verbältnisse. $\ddot{O}_{o}$ bedeutet Reizung mit Oeffnungsschlag oben, $\ddot{O} u$ die gleichartige Reizung unten, $S o$ und $S u$ die entsprechenden Reizungen mit Schliessungsschlägen. \$ bedeutet auf- beziehungsweise absteigende Stromrichtung.

\section{Versuch 1.}

Grasfrosch in üblicher Weise mit dem ganzen Unterschenkel hergerichtet. Platinelektroden. Die ersten Zuckungen durch $\downarrow \ddot{O} \circ$ treten ein bei Verwendung der Thermosäule bei einem Rollenabstand von $13 \mathrm{~cm}$ (R.-A. 13); durch $\uparrow \ddot{O}_{u}$ bei einem Rollenabstand von $12,6 \mathrm{~cm}$. Die Intensitäten dieser beiden Ströme $J$ und $J_{1}$ waren $J=0,5$ und $J_{1}=0,6$, ihr Verhältniss also $1: 1,20$.

Tabellarisch zusammengestellt nimmt dieser Versuch und die ihm ähnlichen folgende Gestalt an.

\begin{tabular}{|c|c|c|c|c|c|c|c|}
\hline \multicolumn{4}{|c|}{ Thermosäule } & \multicolumn{4}{|c|}{ Batterie } \\
\hline & R. A. & $J$ und $J_{1}$ & $\frac{J}{J_{1}}$ & & R.-A. & $J$ und $J_{1}$ & $\frac{J}{J_{1}}$ \\
\hline$\downarrow \ddot{O O}_{0}$ & 13 & 0,5 & 1 & $\downarrow \ddot{O O}$ & 13,3 & 0,557 & 1 \\
\hline$\uparrow \ddot{O} u$ & 12,6 & $\overline{0,6}$ & 1,20 & $\uparrow \ddot{O} u$ & $\overline{13,0}$ & 0,56 & $\overline{1}$ \\
\hline$+S o$ & 7,5 & 7,2 & 1 & $\downarrow S o$ & 12,0 & 0,8 & 1 \\
\hline$\uparrow S u$ & 5,5 & 14,2 & 1,97 & $\uparrow S u$ & $\overline{11,5}$ & 1,05 & 1,28 \\
\hline
\end{tabular}

Versuch 2.

\begin{tabular}{|c|c|c|c|c|c|c|c|}
\hline \multicolumn{4}{|c|}{ Thermosäule } & \multicolumn{4}{|c|}{ Batterie } \\
\hline & R.-A. & $J$ und $J_{1}$ & $\frac{J}{J_{1}}$ & & R.-A. & $J$ und $J_{1}$ & $\frac{J}{J_{1}}$ \\
\hline$\downarrow \ddot{O}_{0}$ & 14,2 & 0,4 & 1 & $\downarrow \ddot{O}_{0}$ & 14,3 & 0,4 & 1 \\
\hline$\uparrow \ddot{O} u$ & 13 & 0,5 & 1,25 & $\uparrow \ddot{O} u$ & 13,5 & 0,5 & 1,25 \\
\hline$\downarrow$ So & 8,5 & 4,8 & 1 & $\downarrow$ So & 13,2 & 0,5 & 1 \\
\hline$\uparrow S u$ & $\overline{6,5}$ & $\overline{10,5}$ & $\overline{2,1}$ & $\overline{\uparrow S u}$ & $\overline{12,2}$ & $\overline{0,8}$ & $\overline{1,4}$ \\
\hline
\end{tabular}




\section{Versueh $\mathbf{3}$.}

\begin{tabular}{|c|c|c|c|c|c|c|c|}
\hline \multicolumn{4}{|c|}{ Thermosäule } & \multicolumn{4}{|c|}{ Batterie } \\
\hline & R.-A. & $J$ und $J_{1}$ & $\frac{J}{J_{1}}$ & & R.A. & $J$ und $J_{1}$ & $\frac{J}{J_{i}}$ \\
\hline$\downarrow \ddot{O O}$ & 14,5 & $\underline{0,38}$ & 1 & $\downarrow \ddot{O O}$ & 14 & 0,41 & 1 \\
\hline$\uparrow \ddot{O} u$ & $\overline{14,5}$ & $\overline{0,38}$ & $\overline{1}$ & $\overline{\uparrow O u}$ & $\overline{13}$ & $\overline{0,57}$ & $\overline{1,4}$ \\
\hline$\downarrow S o$ & 5,5 & 14,2 & 1 & $\downarrow S o$ & 12 & 0,8 & 1 \\
\hline$\overline{\uparrow S u}$ & $\overline{1,5}$ & $\overline{28,4}$ & $\overline{2}$ & $\overline{\uparrow S u}$ & $\overline{11}$ & $\overline{1,2}$ & $\overline{1,5}$ \\
\hline
\end{tabular}

Versuch 4.

\begin{tabular}{|c|c|c|c|c|c|c|c|}
\hline \multicolumn{4}{|c|}{ Thermosäule } & \multicolumn{4}{|c|}{ Batterie } \\
\hline & R. A. & $J$ und $J_{3}$ & $\frac{J}{J_{1}}$ & & R.-A. & $J$ und $J_{1}$ & $-\frac{J}{J_{1}}$ \\
\hline$+\ddot{O O}$ & 14,5 & 0,38 & 1 & $+\ddot{O O}$ & 18 & 0,2 & 1 \\
\hline$\uparrow \ddot{O u}$ & 13,5 & 0,51 & 1,25 & $\uparrow \ddot{O}_{u}$ & 16,5 & 0,26 & 1,3 \\
\hline$+S o$ & 11,7 & 1 & 1 & $\downarrow S o$ & 17,5 & 0,21 & 1 \\
\hline$\uparrow S u$ & 9,2 & 3,3 & 3,3 & $\uparrow S u$ & 16 & 0,26 & 1,23 \\
\hline
\end{tabular}

Da Schliessungsinductionsströme bei Anwendung der Batterie, welche im Verhältniss zu den gleichartigen Strömen bei Anwendung der Thermosäule immer noch sehr schnell verlaufen und auch keinen nennenswerthen Unterschied in ihrer Wirkung oben und unten zeigen (siehe obige Beispiele), so wurden sie weiter nicht beachtet und nur noch mit der Anordnung der Thermosäule einige Versuche angestellt. Einer diene als Beispiel:

Versuch 5.

\begin{tabular}{|c|c|c|c|}
\hline \multicolumn{4}{|c|}{ Thermosäule } \\
\hline & R.-A. & $J$ und $J_{1}$ & $\frac{J}{J_{1}}$ \\
\hline$\downarrow \ddot{O O}$ & 14 & 0,4 & 1 \\
\hline$\uparrow \ddot{\partial u}$ & $\overline{13}$ & $\overline{0,5}$ & 1,25 \\
\hline$\downarrow$ So & 9,3 & 3,2 & 1 \\
\hline$\uparrow S u$ & $\overline{6,8}$ & $\overline{9,7}$ & $\overline{3}$ \\
\hline
\end{tabular}

Stellen wir uns das Ergebniss dieser Versuche und vieler anderer mit gleich artigen, wenn auch nicht durchweg gleichen Ergebnissen, 
graphisch dar, so würden wir etwa folgende schematische Zeichnung erhalten (s. Figur 2). $N$ bezeichnet den in den Muskel $M$ eintretenden Nerven und $\ddot{O}$ über $o$ beziehungsweise $\ddot{O}$ über $u$ die Höhen und den Verlauf der jäh ansteigenden Oeffnungsinductionsströme, welche eben wirksam sind und die ersten Muskelzuckungen auslösen; $S$ über $o$ und $S$ über $u$ dagegen die Höhen und den Verlauf der langsam aufsteigenden Schliessungsinductionsströme, die den gleichen Erfolg auslösen. Man sieht, dass diese letzteren (die in Wirklichkeit viel schneller verlaufen mögen) einmal an und für sich viel höher ansteigen, und dass $S u$ etwa noch einmal so hoch ansteigen muss, als $S o$, um gleich wirksam zu sein (vgl. die fettgedruckten Zahlen in obiger Tabelle).

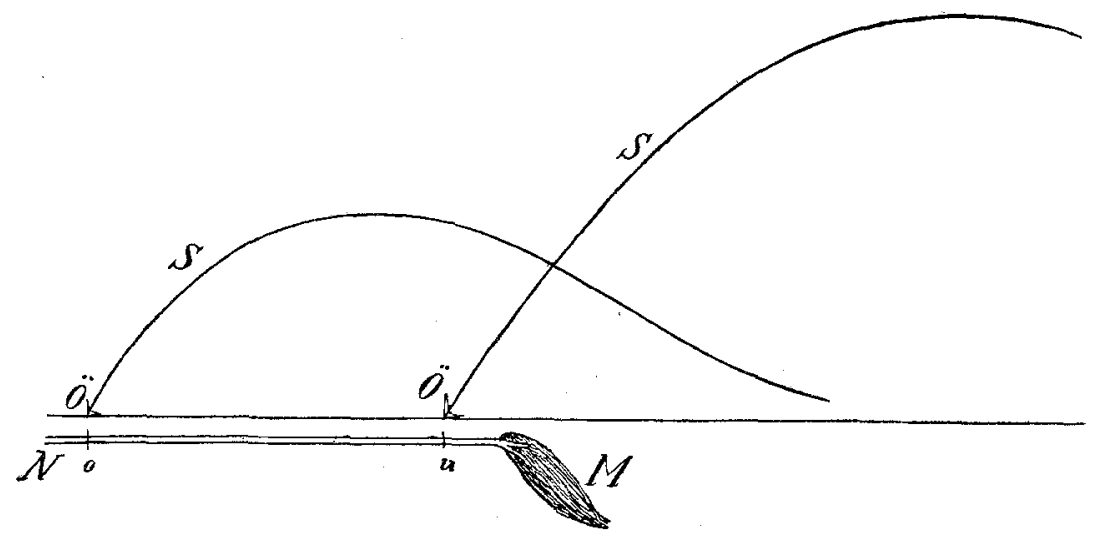

Fig. 2.

Verwendet man Ströme ungünstiger Richtung, also oben aufsteigende und unten absteigende, so zeigen die Versuche (wie übrigens leicht begreiflich) nicht so übereinstimmende Ergebnisse, wie bei günstigen Richtungen. Doch sind auch hier (s. die fettgedruckten Zahlen) die langsam verlaufenden Ströme unten viel weniger wirksam als oben, während die schnell verlaufenden unten gleich oder sogar stärker wirksam sind, als oben.

Zum Belege folgen die Ergebnisse der oben mitgetheilten fünf Versuche, aber bei ungünstiger Stromrichtung. 
Versuch 1.

\begin{tabular}{|c|c|c|c|c|c|c|c|}
\hline \multicolumn{4}{|c|}{ Thermosäule } & \multicolumn{4}{|c|}{ Batterie } \\
\hline & R.-A. & $J$ und $J_{1}$ & $\frac{J}{J_{1}}$ & & R.-A. & $J$ und $J_{1}$ & $\frac{J}{J_{1}}$ \\
\hline$\uparrow \ddot{O O}$ & 11,3 & 1 & 1 & $\uparrow \ddot{O} O$ & 12,8 & 0,5 & 1 \\
\hline $\bar{\downarrow} \dot{\partial u}$ & 12,4 & $\overline{0,6}$ & 0,6 & $\overline{\partial_{u}}$ & 11,5 & 1,0 & $\overline{2}$ \\
\hline$\uparrow S o$ & 3,2 & 22,4 & 1 & $\uparrow$ So & 12,5 & 0,6 & 1 \\
\hline$\downarrow S u$ & $\overline{5,5}$ & $\overline{14,2}$ & 1,63 & $\downarrow S u$ & 13 & $\overline{0,55}$ & $\overline{1,1}$ \\
\hline
\end{tabular}

Versuch 2.

\begin{tabular}{|c|c|c|c|c|c|c|c|}
\hline \multicolumn{4}{|c|}{ Thermosäule } & \multicolumn{4}{|c|}{ Batterie } \\
\hline & R. $-A$. & $J$ und $J_{1}$ & $\frac{J}{J_{1}^{-}}$ & & R. $-A$ & $J$ and $J_{1}$ & $\frac{J}{J_{1}}$ \\
\hline$\uparrow \ddot{O O}$ & 13,8 & 0,4 & 1 & $\uparrow \ddot{O O}$ & 13,5 & 0,5 & 1 \\
\hline$\downarrow \ddot{\partial u}$ & 12,5 & 0,6 & $1, \overline{5}$ & $\downarrow \ddot{O U}$ & 12,2 & 0,8 & 1,6 \\
\hline$\uparrow$ So & 6,9 & 9,5 & 1 & $\uparrow S o$ & 12,5 & 0,6 & 1 \\
\hline$+S u$ & 5 & 16 & 1,7 & $\downarrow S w$ & 11,5 & 1 & 1,7 \\
\hline
\end{tabular}

Versuch 3.

\begin{tabular}{|c|c|c|c|c|c|c|c|}
\hline \multicolumn{4}{|c|}{ Thermosäule } & \multicolumn{4}{|c|}{ Batterie } \\
\hline & R.-A. & $J$ und $J_{1}$ & $\frac{\partial}{J_{1}}$ & & R.-A. & $J$ und $J_{1}$ & $\frac{J}{J_{1}}$ \\
\hline$\uparrow \ddot{\partial}{ }_{0}$ & 12,5 & 0,6 & 1 & $\uparrow \ddot{O O}$ & 10 & 2,1 & 1 \\
\hline$\downarrow \ddot{O} u$ & 14,5 & 0,35 & 0,6 & $\downarrow \ddot{O u}$ & 14 & 0,4 & 0,2 \\
\hline$\uparrow S o$ & 4,5 & 17,9 & 1 & $\uparrow$ So & 10 & 2,1 & 1 \\
\hline$\downarrow S u$ & 6,7 & 27,7 & 1,5 & $\downarrow S u$ & 10,5 & 1,5 & 0,7 \\
\hline
\end{tabular}

Versuch 4.

\begin{tabular}{|c|c|c|c|c|c|c|c|}
\hline \multicolumn{4}{|c|}{ Thermosäule } & \multicolumn{4}{|c|}{ Batterie } \\
\hline & R.-A. & $J$ und $J_{1}$ & $\frac{J}{J_{1}}$ & & R.-A. & $J$ und $J_{1}$ & $\frac{J}{J_{1}}$ \\
\hline$\uparrow \ddot{\partial o}$ & 12,5 & 0,6 & 1 & $\uparrow \ddot{\partial O}_{0}$ & 17,5 & 0,2 & 1 \\
\hline$\downarrow \ddot{O u}$ & 12 & 0,8 & 1,3 & $\downarrow \ddot{O U}$ & 16 & 0,26 & 1,3 \\
\hline$\uparrow S o$ & 11,5 & 1 & 1 & $\uparrow S o$ & 16 & 0,26 & 1 \\
\hline$\downarrow S u$ & 8,7 & 4,3 & 4,3 & $\downarrow S u$ & 15 & 0,3 & 1,2 \\
\hline
\end{tabular}


Versuch 5.

\begin{tabular}{c|c|c|c}
\hline & \multicolumn{3}{|c}{ Thermosäule } \\
\hline & R.-A. & $J$ und $J_{1}$ & $\frac{J}{J_{1}}$ \\
\hline$\uparrow \ddot{O}_{0}$ & $\frac{7,9}{7,8}$ & $\frac{6,4}{6,5}$ & $\frac{1}{1,02}$ \\
$\frac{O_{u}}{\uparrow S o}$ & $\frac{7,7}{6,5}$ & $\frac{6,8}{10,4}$ & $\frac{1}{1,5}$
\end{tabular}

Diese verschiedene Erregbarkeit des Nerven in seinen oberen und unteren Abschnitten gegenüber schnell und langsam verlaufenden elektrischen Reizen gewinnt insofern ein gewisses Interesse, als man sie auch mit dem Leitungsvorgang der normalen Erregung in Verbindung zu bringen veranlasst wird.

Freilich lauten die Angaben hierüber, und zwar selbst über die Verhältnisse am Froschnerven, ganz verschieden. H. Munk ${ }^{1}$ ) und nach ihm Ros enthal ${ }^{2}$ ) behaupteten, dass der Hüftnerv des Frosches in seinen unteren Abschnitten die Erregung langsamer leitet, als in seinen oberen oder dass die Fortpflanzung der Erregung mit verzögerter Gesehwindigkeit vor sich geht. Freilich kommen nach Rosenthal namentlich nach Anlegung eines Querschnittes auch entgegengesetzte Zustände am Hüftnerv des Frosches vor und nach andern Forschern ( $\mathrm{Helmholtz}$ und $\mathrm{Baxt}^{3}$ ) leiten auch die völlig unverletzten Armnerven des Menschen in ihren untern Abschnitten den Reiz langsamer als in ihren oberen; das scheint die Regel zu sein, aber auch entgegengesetzte Angaben werden gemacht, so von Helmholtz und $\mathrm{Baxt}^{4}$ ) und namentlich ron $\mathrm{Place}^{5}$ ).

Die Frage also, ob die Fortpflanzung der Nervenerregung mitgleichmässiger oder mit verzögerter oder mit beschleunigter Geschwindigkeit oder was wohl am wahrseheinlichsten ist, an verschiedenen Stellen der Nerven je nach ihrer Bauart mit verschiedener Geschwindigkeit abläuft, ist noch eine völlig offene. Und es fragt sich, ob etwa aus

1) H. Munk, Archiv für Anat. u. Physiol. 1860. S. 798.

2) J. Rosenthal, Ebenda (Supplement) 1883 S. 240 (274).

3) H. Helmholtz und N. Baxt, Berliner Akademieberichte 1870. Berlin 1871 S. 184 (188).

4) Ebenda 1867. Berlin 1868 S. 228 (234).

5) T. Place, Pflüger's Archiv Bd. 4 S. 424 (435). 1870. 
unseren Versuchen gewisse Anhaltspunkte zur Entscheidung dicser Frage gewonnen werden können.

Die oberen Abschnitte des Hüftnerven, namentlich des Plexus, sind empfindlicher und beweglicher, und dies nicht bloss durch die Nähe des Querschnitts, wie ähnliches sich ja auch aus den Versuchen von Helmholtz und Baxt und von Place für den unverletzten Armnerven des Menschen ergibt. Die unteren sind weniger empfindlich und weniger beweglich und behalten diese Eigenschaften, wie schon vor langer Zeit Pflüger ${ }^{1}$ ) und neuerdings Bürker ${ }^{2}$ ) zeigen konnte, auch bei, wenn man in ihrer Nähe einen Querschnitt anlegt.

Hiernach sollte man, wie auch Grützner in der Efron'schen Arbeit dargelegt hat, vielleicht erwarten, dass der Vorgang der Erregungsleitung immer träger wird oder zum wenigsten in den unteren Abschnitten träger ist. „Es würde hiernach die lebendjge Kraft des physiologischen Reizes (wenn wir uns denselben als rein mechanischen Vorgang denken) jedenfalls eine verschiedenartige, wenn auch nicht nothwendig eine verschieden grosse sein, je nachdem der Reiz in den Muskel oder in das Centrum eintritt. Tritt er in den Muskel, so hätten wir Bewegung grosser Massen mit geringer Geschwindigkeit, tritt er in das Centrum, so hätten wir Eewegung geringerer Massen mit grosser Geschwindigkeit."

Ja es könnten sehr wohl nach der Anschauung von Herrn Prof. Grützner diese "Transformationen" wie bei Leitung der Elektricität sich noch mehrfach im Verlaufe langer Nerven wiederholen. Ob aber, um bei dem Gleichniss zu bleiben, schliesslich in den Endorganen hoch oder niedrig gespannte Ströme, bez. kleine Massen mit grossen Geschwindigkeiten oder grosse Massen mit kleinen Geschwindigkeiten zur Verwendung kommen, oder ob weder das Eine noch das Andere stattfindet, sondern schliesslich nur gewisse mittlere Energieen auf die Endapparate einwirken, darüber wissen wir nichts Näheres.

Beachtenswerth scheint es uns aber immerbin, dass für die unteren Abschnitte die trägen Reize so ausserordentlich vermehrt werden müssen, während bei den schnellen dies nicht nöthig ist. Man wird hier an das "lawinenartige". Anschwellen der Erregung erinnert, ohne dass wir damit sagen wollen, dass thatsächlich unter normalen Verhältnissen in der Nervenfaser während des Fortschreitens der Erregung derartige immer grösser werdende Auslösungen von

1) E. Pflüger, Centralheizung 1859 und Heidenhair, l. e. S. 35.

2) 1. c. S. 37 . 
Spannkräften stattfinden, während "Transformationen" in obigem Sinne sehr wohl vorkommen dürften.

2. Die örtlich verschiedene Erregbarkeit des Hüftnerven bei chemischer Reizung.

Diese Frage ist nicht leicht zu beantworten, weil es namentlich bei starken Salzlösungen, die mạn meistens zu chemischer Reizung der Nerven verwendet, sehr schwierig, wenn nicht unmöglich ist, den Ort der Erregung zu begrenzen. Die entgegenstehenden Angaben von Pflüger auf der einen, sowie von Heidenhain und von v. Fleisch ${ }^{1}$ ) auf der andern Seite, dürften als bekannte Belege hierfür angesehen werden. Wenn man dagegen, wie es Grützner ${ }^{2}$ ) gethan hat, möglichst sorgfältig darauf bedacht ist, einmal nicht gar zu starke Lösungen zu verwenden und zweitens sie an ibrer Ausbreitung über den Nerven zu verhindern, so werden die Frgebnisse durchaus eindeutig und wiederholen sich, wie ich in vielfachen Versuchen gesehen habe, immer in durchaus gleichartiger Weise. Sie ergeben nämlich, dass die unteren Abschnitte des Nerven für chemische Reize ausserordentlich viel bessererregbar sind, als die oberen. Wenigstens fand ich dies bei den von mir angewendeten Flüssigkeiten ausnahmslos. Es ist aber nicht unwahrscheinlich, dass andere reizende Substanzen das gerade Gegentheil von dem ergeben können, was ich gesehen habe; zum mindesten konnte Efron sehen, dass gewisse chemische Stoffe, wie Alkohole, die oberen Abschnitte der Nerven stärker und schneller in ihrer Erregbarkeit beeinflussten, beziehungsweise schädigten, als die unteren.

Ich habe die Grützner'schen Versuche an Hüftnerven, die noch mit dem Rückenmark in Verbindung waren, so wie an solehen, welche hoch oben durchschnitten, beziehungsweise mit einem Faden unterbunden waren, vielfach mit denselben Erfolgen wie Grützner wiederholt. Als reizende Flüssigkeiten verwendete ich NormalKochsalzlösung $(5,84 \%$ ), sowie reines und mit verschiedenen Wassermengen verdünntes Glycerin (l Raumtheil Wasser zu 1 und zu 2 Raumtheilen Glycerin).

1) v. Fleischl, Wiener Akad. Ber. Math. naturw. Kl. Bd. 72. Abth. 3. 1875.

2) Grützner, Pflüger's Archiv Bd. 53 S. 83.1892, 
Der Nerv lag auf einer horizontalen Glasplatte. Die reizende Flüssigkeit befand sich in Fliesspapier, welches in vierfacher Lage in einer Strecke von $1,5 \mathrm{~cm}$ entweder oben oder unten den Nerven verdeckte, während der andere Abschnitt des Nerven mit in physiologische Kochsalzlösung getränktem Fliesspapier bedeckt war.

Statt vieler Versuche seien nur zwei als Belege mitgetheilt.

Versuch, den 9. Juli 1898.

Grosser männlicher Grasfrosch, getödtet 10 Uhr. Nerven mit dem Rückenmark in Verbindung. Reizende Flüssigkeit: Kochsalzlösung von 5,84\%.

\begin{tabular}{|c|c|c|}
\hline Zeit & Reizung : unten & Reizung: oben \\
\hline $\begin{array}{cc}\mathrm{h} & \prime \\
10 & 16\end{array}$ & \multicolumn{2}{|c|}{ Die Fliesspapiere aufgelegt. } \\
\hline 10.18 & \multicolumn{2}{|c|}{$\begin{array}{l}\text { Erste schwache Zuckungen in den } \\
\text { Zehenmuskeln. }\end{array}$} \\
\hline 1019 & \multicolumn{2}{|l|}{$\begin{array}{l}\text { Starke Zuckungen in allen Muskeln. } \\
\text { Ueberwiegen der Beuger. }\end{array}$} \\
\hline 1020 & \multirow{4}{*}{$\begin{array}{l}\text { Beginn des Streckkrampfes. } \\
\left\{\begin{array}{c}\text { Andapernd starker Krampf, nur } \\
\text { ab und zu unterbrochen. }\end{array}\right.\end{array}$} & Leise Zuckungen der Zehen. \\
\hline 1021 & & $\begin{array}{l}\text { Treten stärker auf, aber nicht in } \\
\text { der Weise stark und schnell, } \\
\text { wie bei Reizung unten. }\end{array}$ \\
\hline 1023 & & "Klavierspiel" der Zehen. \\
\hline 1025 & & Zuckungen im Gastrocnemius. \\
\hline 1029 & Tetanus lässt nach. & $\begin{array}{l}\text { Schwacher, abgebrochener Streck- } \\
\text { krampf. }\end{array}$ \\
\hline
\end{tabular}

Versuch, den 10. Juli 1898.

Grosser männlicher Grasfrosch, getödtet 11 Uhr. Nerven hoch oben unterbunden und durchschnitten. Sonst die gleiche Anordnung. Reizende Flässigkeit $\mathrm{NaCl} 5,84 \%$.

\begin{tabular}{|c|c|c|}
\hline Zeit & Reizung: unten & Reizung: oben \\
\hline b 11 & \multicolumn{2}{|c|}{ Auflegen der Fliesspapierbäusche. } \\
\hline 1115 & Zuckungen der mittleren Zehe. & \\
\hline $\begin{array}{ll}11 & 16 \\
11 & 17\end{array}$ & $\begin{array}{l}\text { Alsbald betheiligt sich der Fuss. } \\
\text { Ganzer Schenkel in klonischem } \\
\text { Tetanus. }\end{array}$ & $\begin{array}{l}\text { Leises, seltenes Zucken der Mittel- } \\
\text { zehe und des Fusses. }\end{array}$ \\
\hline 1118 & Uebergehen jn Streckkrampf. & $\begin{array}{l}\text { Das ganze Bein geräth in ruck- } \\
\text { weise Bewegung. }\end{array}$ \\
\hline 1119 & Streckkrampf: & Zuckungen des Gastroenemius. \\
\hline 1120 & 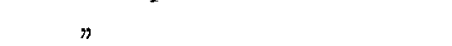 & Werden stärker. \\
\hline 1122 & $"$ & Klonischer Tetanus. \\
\hline 1124 & Lässt nach. & $"$ \\
\hline 1125 & $\begin{array}{l}\text { Versuch beendet. Ein eigentlich } \\
\text { nicht zu Stande gekommen. }\end{array}$ & r Tetanus war bei Reizung oben \\
\hline
\end{tabular}


Auch die mit anderen reizenden Flüssigkeiten (Glycerin) angestellten Versuche ergaben durchweg gleichartige Resultate. Wurde der Nerv unten gereizt, so traten in der Regel die Zuckungen in. den Muskeln viel früher ein und entwickelten sich stets zu vollkommeneren, kräftigeren und länger anhaltenden Krämpfen, als dies. bei Reizung oben der Fall war.

Sehr dentlich zeigen dies auch graphische Aufzeichnungen dieser Vorgänge bei alleiniger Verwendung des Wadenmuskels. Es war eine Einrichtung derart getroffen, dass auf sehr langsam sich drehender Walze die beiden mit geringen Gewichten $(5 \mathrm{~g})$ beschwerten Muskeln ihre Zuckungen in etwa 3,5 facher Vergrösserung übereinander aufschreiben konnten. Die Nerven lagen auf horizontalen: Glasplatten, der eine oben, der andere unten in gleicher Ausdehnung von 1,5-1,8 cm mit dem Fliesspapier bedeckt, welches mit der reizenden Flüssigkeit getränkt war. Um möglichst jedes Ausbreiten der Flüssigkeiten längs des Nerven zu verhindern, war der Nerv etwa in seiner Mitte in einen Vaselindamm eingebettet, auf dessen einer Seite sich also das Fliesspapier mit der reizenden Flüssigkeit, auf dessen anderer Seite sich das mit physiologischer Kochsalzlösung getränktè Fliesspapier befand. Auf diese Weise war es möglich, zu zeigen, dass die oberen Abschnitte des Nerven viel später, manchmal eine Viertelstunde später erregt wurden, als die unteren, und dasszweitens die Erregungen oben einen ganz anderen Charakter haben, als diejenigen unten.

Die beifolgenden Curven erläutern die Verhältnisse. Figur 1 auf Taf. I zeigt die Einwirkung von verdünntem Glycerin (2 Glycerin: 1 Wasser). Die Zuckungen in Folge der Reizung oben, welche in der oberen Curve dargestellt sind, treten später, etwa eine Minute später, ein und erreichen nicht die Höhe wie diejenigen, welche in Folge der Reizung unten erzeugt wurden. Die (grösstentheils nachträglich eingezeichneten) Zeitmarken bedeuten durchweg Minuten.

In Figur 2 Taf. I, bei Anwendung von schwächerem Glycerin (1 Glycerin: 1 Wasser), ist der Unterschied noch auffälliger. Die Reizung oben macht sich in der Curve etwa erst 18 Minuten nach dem Bedecken der Nerven mit der reizenden Flüssigkeit bemerkbar bei $A$. 3 Minuten vorher waren allerdings schon an dem Muskel selbst schwache Zuckungen zu sehen, die sich jedoch nicht auf den Zeichenhebel übertrugen. Auch die späteren Zuckungen sind ungemein schwach und zeigen kaum Summationen zu einem Tetanus. Bei 
$\underset{\times}{\times}$ wurde die Trommel angehalten und erst wieder in Bewegung gesetzt, als diese ganz schwachen, in der Curve nicht sichtbaren Zuckungen begannen.

Ganz anders verhält es sich mit der Reizung der unteren Abschnitte des Nerven. Acht Minuten nach Beginn der Reizung traten zunächst geringfügige Zuckungen auf, welche sich aber bald, nach etwa einer Minute, zu immer kräftigeren tetanischen Zusammenziehungen entwickeln und später nahezu glatten, nur von einzelnen Zuckungen unterbrochenen Tetanis Platz machen.

Noch sei auf eine merkwürdige Erscheinung hingewiesen, die wir bei Anwendung schwacher Reize auf die oberen Nervenabschnitte niemals vermissten. Es zeigte sich nämlich durchweg; dass, ehe die Zusammenziehungen begannen, und auch häufig nach den ersten schwachen Zusammenziehungen, sich der Muskel verlängerte (s. Fig. 3 bei $A$ Taf. I). Es war also im Anfang des Versuches eine grössere Spannung in demselben vorhanden, als nach Einwirkung dieses schwachen Reizmittels auf seinen Nerven. Dasselbe hatte den in den eigentlichen motorischen Nerven befindlichen Tonus herabgesetzt, oder, was im Erfolge auf das Gleiche hinauskommt, seine Erschlaffungsnerven, die man wohl auf Grund der Untersuchungen von Grützner

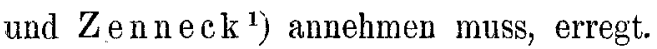

Diese eigenthümliche Dehnung des Muskels, sowie die ganz verschiedene Erregung desselben von den oberen, beziehungsweise unteren Nervenstellen aus, zeigen schliesslich noch die letzten von mir mitgetheilten Curven in Fig. 3 Taf. I. Zur Verwendung kam Glycerin, mit Wasser zu gleichen Theilen versetzt. Die Zuckungen bei Reizung unten begannen nach einer Minute, diejenigen bei Reizung oben nach $61 / 2$ Minuten und von einer viel niedrigeren Abscisse, als dem Muskel im Beginn des Versuches zukam. Hierauf blieben sie etwa $3 \frac{1}{2}$ Minuten aus und traten erst dann, und zwar wesentlich in Form von einzelnen Zuckungen, nicht in Form von Tetanis auf. Man sieht also, wie auch hier das hemmende, sozusagen negative Moment erfolgreich mit dem fördernden, positiven kämpft, das bei Reizung unten in den Vordergrund tritt.

Erwähnt sei schliesslich noch, dass die Durchschneidung des Nerven in seiner Mitte bei Reizung oben den Muskel vollständig zur Ruhe brachte, dass also die reizende Flüssigkeit sich nicht auf die unteren Stellen ausgebreitet hatte.

1) G. Zenneck, Pflüger's Archiv Bd. 76 S. 21. 1899. 
Ich wiederholte ferner auch noch die Versuche von Groves und fand wie er, dass, wenn man die auseinandergebreiteten oberen Plexustheile des Nerven oder gar die motorischen Wurzeln vertrocknen lässt, der Muskel nicht oder doch viel schwächer und später zuckt, als wenn man die unteren Abschnitte vertrocknen lässt. Selbstverständlich müssen diejenigen Nervenabschnitte, welche nicht vertrocknen sollen, in zweckmässiger Weise vor Vertrocknung geschützt sein.

Die Frage dieser ausserordentlich verschiedenen Erregbarkeit des Nervenstammes bei chemischer Reizung oben oder unten ist schwer zu beantworten. Grützner bringt sie, an Untersuchungen von ihm ${ }^{1}$ ) und Clara Halpers $0 \mathrm{n}^{2}$ ) erinnernd, mit deren verschiedenem, anatomischem Bau in Beziehung, worauf hier nur flüchtig hingewiesen sei. Wenn nun auch aus irgend welchen Ursachen, sei es in Folge des besseren Eindringens der Flüssigkeit zum Achsencylinder, sei es in Folge stärkerer Pressung bei Reizung der unteren bindegewebsreicheren Abschnitte, die Erregung unten stärker ist, als oben, so bleibt es doch unverständlich, wie dasselbe Reizmittel oben entschieden hemmend, unten erregend wirkt.

Gelegentlich prüfte ich auch die Wirkungen schwacher Kalisalzlösungen auf den Nerven und fand, wie dies bereits von anderer Seite, namentlich von Biedermann${ }^{3}$ ) des Genaueren gezeigt wurde, zunächst eine erhöhte Erregbarkeit gegenüber Schliessung und Oeffnung elektrischer Ströme, dann aber bei gleichzeitigem Sinken der Erregbarkeit eine, kurz gesagt, immer stärkere Wirkung des physiologischen Anelektrotonus. Es traten schon bei verhältnissmässig schwachen Strömen Oeffnungszuckungen auf und bei ebenfalls ziemlich schwachen Strömen versehwanden die Schliessungszuckungen aufsteigender Ströme vollkommen.

Führt man z. B. den Pflüger'schen Versuch aus, um die verschiedene Erregbarkeit an anelektrotonischen und katelektrotonischen Strecken des Nerven nachzuweisen, so kann man sich mit Leichtigkeit davon uberzeugen, dass in der ersten Zeit der Einwirkung einer ${ }_{\omega_{\mathrm{D}} \mathrm{s}}^{\mathrm{s}} \mathrm{chwachen}$ Kalisalzlösung auf den Nerven ausserordentlich viel schwächere Ströme nothwendig sind, um die gleichen, sowohl erreg-

1) S. oben S. 158 (S. 47 der genannten Arbeit).

2) S. ebenda S. 158.

3) W. Biedermann, Elektrophysiologie 1895 S. 593. 
barkeitserhöhenden, wie herabsetzenden Wirkungen hervorzubringen, als unter normalen Verhältnissen.

Nach längerer Einwirkung des Salzes dagegen macht sich nur die stärkere Wirkung des physiologischen Anelektrotonus bemerkbar, diejenige des Katelektrotonus dagegen ist bedeutend herabgesetzt, l. b. es bedarf immer noch ausserordentlich schwacher Ströme, um die Erregbarkeit auffällig herabzusetzen, dagegen sehr viel stärkerer Ströme, um sie zu erhöhen. Ein Versuchsbeispiel wird genügen.

\section{Versuch.}

6 kleine Daniells liefern den polarisirenden Strom, der durch ein Si emen s'sches. Rheochord in der Nebenleitung in bekannter Weise durch Stöpselung verändert werden kann. Den reizenden Strom liefert ein gewöhnlicher Inductionsapparat. Grosser männl. Grasfrosch getödtet 11 Uhr. Pol. Elektroden oben, reizende unten. $11^{\text {h }}$ 09'. Die starke Oeffo.-Ind.-Zuckung (Oe.J.Z.) bei Rollenabstand (R.-A.) $18 \mathrm{~cm}$ verschwindet durch Strom $\uparrow$ von Stärke $76^{1}$ ).

Die eben merkbare Oeff.-Ind.-Zuck. bei R.-A. $21 \mathrm{~cm}$ wird kräftig durch Strom $\downarrow$ von Stärke 15 .

Der ganze Nerv 2 Minuten lang in $\frac{1}{10} \mathrm{n}$. KCl $(0,74 \%)$ gelegt.

$11^{\mathrm{h}}$ 15'. Starke Oe.J.Z. bei R.-A. $19 \mathrm{~cm}$ verschwindet durch Strom $\uparrow$ von der Stärke 4.

Ëben merkbare Oe.J.Z. bei R.-A. $21 \mathrm{~cm}$ wird kräftig durch Strom von der Stärke 9.

Der Nerv wird 5 Minuten in physiolog. Kochsalzlösung ausgespüit.

11 h $25^{\prime}$ treten die entsprechenden Erscheinungen bei R.-A. $18 \mathrm{~cm}$ auf durch Strom $\uparrow$ von der Stärke 14,5, und bei R.A. $20 \mathrm{~cm}$ auf durch Strom $\downarrow$ von der Stärke 9,2.

Der Nerv wird wieder in die KCl-Lösung eine Ninute lang gelegt.

11 h 32 ' erhält man bei R.-A. $18 \mathrm{~cm}$ die bemmende Wirkung durch Strom $\uparrow$ von der Stärke 11, und bei R.-A. $21 \mathrm{~cm}$ die erhöhende Wirkung durch Strom $\downarrow$ von der Stärke 70 .

Auch die Wirkung schwacher Alkohollösungen (1 Alkohol auf 100 physiologische Kochsalzlösung) prüfte ich gelegentlich. Bekanntlich erböht Alkohol, in dieser Art auf Nerven angewendet, ihre Erregbarkeit. Er versetzt sie aber auch, wenn ich so sagen darf, in eine dauernde Unruhe, in ein fortwährendes inneres Erzittern, welches sich zwar ohne Weiteres in keiner Weise in dem Muskel bemerkbar macht, wohl aber - worauf mich Herr Prof. Gr ützner hinwies - sehr hübsch in folgender Weise gezeigt werden kann.

1) Das heisst 76 Siemen seinheiten im Nebenschluss. 
Biedermann ${ }^{1}$ ) hat, an frühere Angaben von Pflüger anknüpfend, die wohl jedem mit elektrischen Reizversuchen Vertrauten bekannte verspätete Oeffnungserregung des Genaueren untersucht und unter Anderem die interessante Angabe gemacht, dass alkoholisirte Nerven bei elektrischer Reizung ausserordentlich sehön in dem zugehörigen Muskel diese verspätete (und auch die normale) Oeffnungserregung zeigen. Die erste, sofort nach der Oeffnung des Stromes einsetzend, ist kurz, die zweite, später auftretende dagegen gedehnt, tetanisch. Wenn man nun diese verspätete Oeffnungerregung nicht an einem einzelnen Muskel, sondern an der ganzen unteren Extremität beobachtet, so sieht man einige Zeit nach Oeffnung des den Nerven reizenden. Stromes ein lang dauerndes Zappeln und Bewegen aller möglichen Muskeln, namentlich auch derjenigen der Zehen, die sich fast wie die Finger eines Klavierspielers bewegen, und welche auf das Schönste die lange Zeit andauernden und nach der Oeffnung des Stromes wirksam werdenden einzelnen Reizanstösse zeigen.

3. Die örtlich verschiedene Erregbarkeit des Hüftnerven gegenüber mechanischer Reizung.

Es ist bekannt, dass die verschiedensten mechanischen Einwirkungen von der gewaltsamen Zertrümmerung oder Zerquetschung des Nerven bis zu einer ganz leichten Erschütterung desselben, wie sie von einem leichter auf ihn auffallenden Hämmerchen oder Gewicht ausgeübt wird, den Nerven reizt und sein Endorgan ebenfalls in Erregung versetzen kann.

Indem ich betreffs der älteren Literatur dieses Gegenstandes auf die bekannte, eingehende Studie von Tigerstedt ${ }^{2}$ ) verweise, welcher zuerst den mechanischen Reiz genau seiner Grösse nach maass, sei hier nur mitgetheilt, dass nach Tigerstedt sich, so viel mir bekannt, Hällstén ${ }^{3}$ ), Langendorff ${ }^{4}$ ), Piotrowski ${ }^{3}$ ) und v. Uexkülls) mit der mechanischen Nervenreizung beschäftigt haben.

1) Elektrophysiologie S. 587.

2) R. Tigerstedt, Studien über mechan. Nervenreizung. Helsingfors 1880 .

3) K. Hällstén, Archiv für (Anat. u.) Physiolog. 1881 S. 90 u. G. Piotrowski. Ebenda 1898 S. 205 (273).

4) 0. Langend orff, Centralbl. f. d. med. Wissensch. 1882 S. 113.

5) J. v. Uexküll, Zeitschrift für Biologie Bd. $31 \mathrm{~S} .148,1895$ und $\mathrm{Bd} .32$ S. 438,1895 . 
Tigerstedt liess versehieden schwere Gewichte, meistens eines von $0,485 \mathrm{~g}$, versehieden hoch $(1,6-7,4 \mathrm{~mm})$ auf den Nerven herabfallen, indem die durch einen Elektromagneten festgehaltenen Gewichte durch eben denselben losgelassen wurden und in einer Gleitvorrichtung senkrecht auf den Nerven herabfielen. Später bediente $\mathrm{rr}^{1}$ ) sich auch eines kleinen elektromagnetischen Hämmerchens, ähnlich wie dies Heidenhain zuerst mit einem Wagner'schen Hammer gethan hatte. Nur konnte die Schlagkraft des Tigerstedt'schen Hämmerchens ausserordentlich fein abgestuft werden.

Langendorff zeigte, dass eir durch eine schwingende Stimmgabel mit einem Faden verbundener Nerv kräftig tetanisch gereizt werden kann; Piotrowski, der unter Gads Leitung arbeitete, beschrieb einen mechanischen Reizapparat, der mit der Handbabung des von uns angewendeten eine gewisse Aehnlichkeit hat, und v. Uexk üll schliesṣlich stellte die verschiedensten Möglichkeiten zulsammen, die alle mechanisch reizend auf einen Nerven wirken. Auch entdeckte er die interessante Thatsache, dass Entlastung eines Nerven reizend auf denselben wirken kann. Er führt allerdings diese Reizung, die auch ich hin und wieder beobachtete, auf elektrische Reizung zurück.

Von besonderem Interesse aber ist für uns die Arbeit von Hällstén, weil dieser Forscher sich eines ganz eigenen Reizapparates bediente und $\mathrm{zu}$ andern Anschauungen als Tigerstedt betreffs der örtlichen Erregbarkeit des Hüftnerven kam. Tigerstedt gibt nämlich an, dass "die Irritabilität für mechanische Reize an einem jeden Punkte des Nerven gleich gross ist". Hällstén dagegen findet ähnlich wie Pflüger durchweg die grössere Erregbarkeit an den centralen Stellen des Nerven. Seine hübsche Methode, die allerdings keine so genaue Messung wie diejenige von Tigerstedt zuliess, bestand darin, dass eine mehr oder weniger gehobene Elfenbeinkugel anf eine Marey'sche Trommel fiel, und dass der Zeichenhebel einer zweiten, mit dieser in Verbindung stehenden Trommel auf den Nerven schlug und ihn mehr oder weniger stark reizte.

Der von mir verwendete Reizapparat, den mir Herr Prof.

1) Beiträge zur Physiologie, C. Ludwig zu seinem 70. Geburtstage gewidmet. Leipzig 1887 S. 82. 
Grützner hatte herrichten lassen, bestand im Wesentlichen aus einem leichten Elfenbeinbämmerchen, welches mehr oder weniger beschwert werden und von verschiedener Höhe auf den Nerven fallen konnte. Der Nerv selbst lag gerade gestreckt auf einer horizontalen Glasplatte, welche mit zwei Lagen von feuchtem, in physiologische Kochsalzlösung getränktem Fliesspapier belegt war. Auf diese Weise ${ }^{1}$ ) wurde einmal der Nerv in seiner Lage festgehalten, so dass das Hämmerchen ihn immer genau mit seiner Mitte traf, andrerseits wurde eine Vertrocknung des Nerven durch Feuchthalten des Fliesspapiers vermieden.

An der einen langen Seite der den Nerven in ihrer Mitte tragenden Glasplatte befand sich eine in Millimeter getheilte Gleitschiene, an welcher das Hämmerchen verschoben und stets so festgestellt werden konnte, dass, es horizontal stehend, gerade auf den Nerven zu liegen kam. So war man sicher, stets dieselben oder doch nahezu dieselben Stellen des Nerven mit gleicher Kraft zu treffen, wenn man den Fallapparat an dieselben Orte der Gleitschiene einstellte, und konnte so den Nerven an versehiedenen Stellen seines Verlaufes, gewöhnlich oben, mitten und unten reizen.

Das Hämmerchen selbst war ausserordentlich Jeicht gearbeitet, und spielte in einem feinen horizontalen Achsenlager. Der Stiel desselben war entweder ein dünner Aluminiumdraht von etwa $1 \mathrm{~mm}$ Durchmesser und $9 \mathrm{~cm}$ Läuge oder ein $10 \mathrm{~cm}$ langer steifer Grashalm. $9 \mathrm{~cm}$ von der Achse befand sich das kleine Elfenbeinhämmerchen, welches nach oben einen leichten kleinen Aluminiumstiel trug, auf den man kleine runde, in der Mitte durchbohrte Messing- oder Bleigewichte aufschieben konnte. Das Hämmerchen selbst war 4,5 mm lang, 2,5 mm dick und $4 \mathrm{~mm}$ hoch. Die Schlagfläche war abgerundet und stand natürlich sowie der Stiel des Hämmerchens senkrecht zur Richtung des Nerven.

Um nun das Hämmerchen verschieden boch herabfallen zu lassen, befand sich neben seinem Stiel ein Gradbogen und in ihm Löcher, die in bestimmten Höhen durch denselben gebohrt waren und einen Stift aufnehmen konnten. Hob man den Stiel des Hämmerchens bis an den über ihm stehenden Stift herauf, so wusste man die Höhe, von

1) Dieser kleine Kunstgriff wurde, wie ich erst später sah, auch von Tiger stedt in seinem neuen Apparat angewendet. 
welcher das Hämmerchen herabfiel. Gewöhnlich kamen nur 4 Stiftstellungen, beziehungsweise 4 verschiedene Fallhöhen von $1-4 \mathrm{~cm}$ zur Verwendung.

Besondere Sorgfalt wurde nun der Auslösung des fallenden Hämmerchens zugewendet. Sie geschah folgendermaassen. Der Stiel des Hämmerchens war nach hinten über seine Achse hinaus um nahezu $3 \mathrm{~cm}$ verlängert. Nahe am Ende dieser rückwärtigen, durchaus steifen Verlängerung waren zwei kleine, $2 \mathrm{~mm}$ von einander entfernte Korkwürfelchen auf sie aufgeschoben und befestigt. Senkrecht zu der Drehungsachse des Hämmerchens und ebenfalls horizontal gestellt befand sich die Achse eines zweiarmigen Hebels, dessen eines Ende in eine Schweinsborste von etwa 6-8 $\mathrm{mm}$ Länge endete. Drehte man diesen Hebelarm abwärts, so drückte die Schweinsborste auf den nach hinten verlängerten Stiel des Hämmerchens und legte sich immer genau an dieselbe Stelle an eine Fläche des Korkwürfelchens an. Zugleich bob sie den Hammer bis an den Arretirungsstift. Drehte man ein wenig weiter, so bog sich die Borste durch und der Hammer fiel auf den Nerven. (Diese Einrichtung hat eine gewisse Aehnlichkeit mit dem in der Arbeit von Piotrowski in Taf. $X$ abgebildeten Reizapparat, auf den hiermit verwiesen sei. Nur befand sich bei uns der Auslösungshebel nicht in derselben vertikalen Ebene, wie der fallende Hammer, sondern war senkrecht zu derselben gestellt.) Drehte man den zweiarmigen Hebel mit der auslösenden Borste weiter, so ging das kürzere andere Hebelende an dem verlängerten Hammerstiel vorbei und die Borste kam wieder über diesen zu stehen, um bei weiterer Drehung ihn wieder herabzudrücken, beziehungsweise den Hammer bis an den Arretirungsstift zu heben. Auf diese Weise konnte man in beliebigen Zwischenräumen und, wenn nöthig, auch ziemlich schnell hintereinander gleiche Reize auf dieselbe oder auf verschiedene Stellen des Nerven einwirken lassen.

Dass die Schläge gleich stark waren, davon überzeugten wir uns nach der Methode von Hällstén, indem wir den Nerven durch eine empfindliche Marey'sche Trommel ersetzten, welche mit einer ebensolchen Zeichentrommel verbunden war.

Die Ausschläge des Zeichenhebels der zweiten Trommel, welcher auf einen berussten Cylinder zeichnete, fielen unter denselben Versuchsbedingungen durchaus gleich aus. Geringe Veränderungen der Hubhöhe des Hammers, beziehungsweise solche des Gewichtes ver- 
änderten den Ausschlag in deutlich sichtbarer Weise nach der einen oder andern Richtung:

Auch zeichnete ich hin und wieder die Fallcurven des Hammers, der mit einem feinen Härchen versehen war, auf einem schnell rotirenden Cylinder auf, und bestimmte so die Endgeschwindigkeit des fallenden Hammers beim Aufschlag auf die Unterlage. Erst später fand ich, dass Tigerstedt seinen neuen Apparat in ganz ähnlicher Weise durch Bestimmung der Fallzeiten seines Hämmerchens ausgewerthet hat.

Ganz sicher konnte ich also sagen, dass unter gleichen Versuchsbedingungen unser Apparat (natürich innerhalb gewisser Fehlerquellen) auch durchaus gleiche Reize lieferte. Eine andere, später zu erörternde Frage ist die, ob und unter welchen Bedingungen derselbe bei verschiedenen Hubhöhen, beziehungsweise Gewichten auch gleiche Energiemengen lieferte.

Die erste Frage, an deren Beantwortung ich heranging, war die, ob der Nerv an den verschiedenen Stellen gegenüber mechanischen Reizen die gleiche Erregbarkeit zeigte. Hierbei kam ich nun an frischen Nerven ausnahmslos zu denselben Ergebnissen wie Hällstén. Immer war der Nerv oben erregbarer als unten; dies galt sowohl für den undurchschnittenen, mit dem Rückenmark in Verbindung stehenden, wie namentlich für den hoch oben durebschnittenen und mit einem Faden unterbundenen Nerven.

Es mögen folgende Versuche als Belege dienen.

\section{Versuch.}

Mittelgrosser männlicher Grasfrosch, getödtet $9 \mathrm{~h} 35^{\prime}$. Hüftnerven hoch oben mit einem Faden unterbunden und oberhalb des Fadens durchschnitten. Hierauf das Nervmuskelpräparat in physiologischer Kochsalzlösung abgespült.

\begin{tabular}{|c|c|c|c|c|c|c|}
\hline \multirow{2}{*}{$\begin{array}{l}\text { Nummer } \\
\text { des } \\
\text { Versuches }\end{array}$} & \multirow{2}{*}{ Zeit } & \multirow{2}{*}{ Fallhöhe } & \multirow{2}{*}{$\begin{array}{c}\text { Belastung } \\
\text { des } \\
\text { Hammers } \\
\text { in gr }\end{array}$} & \multicolumn{3}{|c|}{ Zuckungshöhe in $\mathrm{mm}$} \\
\hline & & & & oben & mitten & unten \\
\hline $\begin{array}{l}1 \\
2 \\
3\end{array}$ & $\begin{array}{lc}\text { hl } & \text { ' } \\
9 & 45 \\
9 & 48 \\
9 & 54\end{array}$ & $\frac{20^{\circ}}{-}$ & $\frac{0}{-}$ & $\begin{array}{l}7,0 \\
8,5 \\
6,0\end{array}$ & $\begin{array}{l}5,0 \\
5,7 \\
2,5\end{array}$ & $\begin{array}{l}0,5 \\
5,0 \\
1,0\end{array}$ \\
\hline \multicolumn{7}{|c|}{ Nerv voṛ Vertrocknung geschützt, ruhig liegen gelassen } \\
\hline $\begin{array}{l}4 . \\
5 \\
6\end{array}$ & $\begin{array}{ll}10 & 10 \\
10 & 14 \\
10 & 17\end{array}$ & $\frac{10^{\circ}}{20^{\circ}}$ & $\begin{array}{l}0,255 \\
-\end{array}$ & $\begin{array}{l}5,0 \\
0,0 \\
1,0\end{array}$ & $\begin{array}{l}6,5 \\
6,0 \\
1,5\end{array}$ & $\begin{array}{l}8,0 \\
4,5 \\
6,0\end{array}$ \\
\hline
\end{tabular}


Aus diesem Versuch, dem ich viele andere gleichartige anschliessen könnte, geht zunächst hervor, dass der frische Nerv oben, d. h. etwa in der Mitte des Plexus, viel erregbarer ist als in seiner Mitte und namentlich unten nahe dem Muskel. Die Erregbarkeit nimmt hier mit der Zeit $a b$, und später sind häufig nach einem Stadium von nahezu durchweg gleicher Erregbarkeit (Versuch 4) gemäss den Ritter-Valli'schen Gesetz die unteren Stellen besser erregbar, als die oberen.

Bei all' diesen Versuchen machten wir nebenher dieselben Beobachtungen wie Tigerstedt. Namentlich konnten wir uns auch davon überzeugen, dass leichte mechanische Reizungen die Erregbarkeit für bald darauf folgende Reize steigern. Es wurde deshalb auch immer längere Zeit zwischen den einzelnen Reizungen gewartet. Der Hammer war, was nicht unwichtig ist, während dieser Zeit vom Nerven abgehoben, sonst traten leicht die v. Uexküll'schen Entlastungszuckungen ein.

Da die Zuckungshöhen aus diesen und andern Gründen (man traf auch nicht immer ganz genau dieselbe Stelle des Nerven) unter sonst gleichen Bedingungen nicht immer ganz gleich ausfielen, so mögen hier noch einige Versuche an durchschnittenen Nerven mitgetheilt sein, in denen mehrfache Reizungen hintereinander ausgeführt wurden, gewöhnlich 5. Es sind dann die mittleren Höhen, d. h. das arithmetische Mittel aus den 5 Zuckungen, sowie die grösste und kleinste angegeben. Bald wurde oben, bald unten zuerst gereizt.

\section{Versuch 1, den 6. Juli 1898.}

Ziemlich grosser männlicher Grasfrosch, getödtet gegen $10^{\mathrm{h}}$. Nerven durchschnitten und, wie immer, mit einem Faden abgebunden.

\begin{tabular}{|c|c|c|c|c|c|c|c|c|c|c|c|c|}
\hline \multirow{3}{*}{ 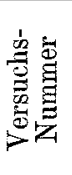 } & \multirow[b]{3}{*}{ 焉 } & \multirow{3}{*}{ 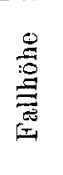 } & \multirow{3}{*}{ 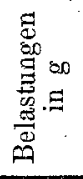 } & \multicolumn{9}{|c|}{ Zuckungshöhe in Millimetern } \\
\hline & & & & \multicolumn{3}{|c|}{ Oben } & \multicolumn{3}{|c|}{ Mitten } & \multicolumn{3}{|c|}{ Unten } \\
\hline & & & & 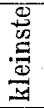 & $\begin{array}{l} \\
0 \\
0 \\
0 \\
0 \\
0 \\
50\end{array}$ & 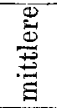 & 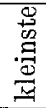 & 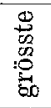 & & 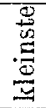 & 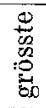 & 总 \\
\hline 1 & 10,05 & $20^{\circ}$ & 0 & 10 & 10 & 10 & 0 & 0 & 0 & 0 & 0 & 0 \\
\hline 2 & 10,08 & - & 0,255 & 10 & 13 & 11 & 8 & 12 & 9,5 & 2 & 7 & 5,2 \\
\hline 3 & 10,14 & - & - & 10 & 11,5 & 10,7 & 9,5 & 10 & 9,7 & 5 & 8 & 7,1 \\
\hline
\end{tabular}


Versuch 2, den 7. Juli 1898.

Grosser weiblicher Grasfrosch, getödtet 11 h 18'. Nerven durchschnitten.

\begin{tabular}{|c|c|c|c|c|c|c|c|c|c|c|c|c|}
\hline \multirow{3}{*}{ 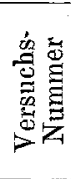 } & \multirow[b]{3}{*}{$\begin{array}{l}\stackrel{\mathscr{C}}{\mathrm{J}} \\
\end{array}$} & \multirow{3}{*}{ 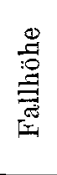 } & \multirow{3}{*}{ 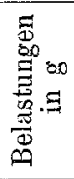 } & \multicolumn{9}{|c|}{ Zuckungshöhe in Millimetern } \\
\hline & & & & \multicolumn{3}{|c|}{ oben } & \multicolumn{3}{|c|}{ Mitten } & \multicolumn{3}{|c|}{ Unten } \\
\hline & & & & 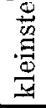 & 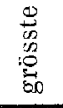 & 总 & 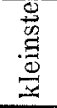 & 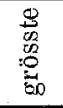 & 离 & 䓯 & 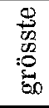 & 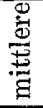 \\
\hline 1 & 11,26 & $20^{\circ}$ & 0 & 9,4 & 11,9 & 10,3 & 4,5 & 6,9 & 5,9 & 0 & 0 & 0 \\
\hline 2 & 11,29 & - & - & 9,6 & 11,0 & 10,04 & 9 & 11 & 10,4 & 0 & 0 & 0 \\
\hline 3 & 11,9 & 一 & - & 6 & 7 & 6,4 & 3 & 8 & 5,1 & 0 & 0 & 0 \\
\hline
\end{tabular}

Versuch 3, den 7. Juli 1898

an einem mittelgrossen Männchen, getödtet $12^{\mathrm{h}}$. Nerven durchschnitten.

\begin{tabular}{l|l|l|l|l|l|l|l|l|l|l||l|l|l}
1 & 12,10 & 200 & 0 & 11 & 12 & $\mathbf{1 1 , 6}$ & $\mathbf{7 , 9}$ & 9 & $\mathbf{8 , 6}$ & 0 & 0 & $\mathbf{0}$ \\
2 & 12,13 & - & - & 12 & 14 & $\mathbf{1 2 , 7}$ & $\mathbf{7 , 5}$ & 8 & $\mathbf{7 , 6}$ & 0 & 0 & 0 \\
3 & 12,16 & - & - & 12 & 15 & $\mathbf{1 2 , 8}$ & 8 & 12 & $\mathbf{9 , 9}$ & 4,5 & $\mathbf{7}$ & $\mathbf{5 , 6}$
\end{tabular}

Abgesehen von geringen Unregelmässigkeiten geht aus allen diesen Versuchen hervor, dass der (durchschnittene) Nerv oben erregbarer ist, als unten.

Um die Wirkung des Quersehnittes möglichst zu beseitigen, liess ich bei den folgenden Versuchen die Nerven im Zusammenhang mit dem Rückenmark. Aber auch diese Maassnahme änderte die Frgebnisse nicht wesentlich, wie die folgenden Versuche beweisen.

Versuch, den 8. Juli 1898.

Vorbereitungen wie früher angegeben.

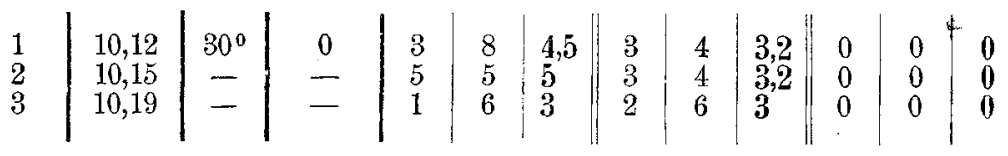

Versuch, den 12. Juli 1898.

\begin{tabular}{l|l|l|l|l|l|l||l|l|l||l|l|l}
$\mathbf{1}$ & 10,15 & $30^{\circ}$ & 0 & 2 & 7,5 & $\mathbf{4 , 5}$ & 3 & 3 & $\mathbf{3}$ & 0 & 0 & $\mathbf{0}$ \\
$\mathbf{2}$ & 10,18 & - & - & 5 & 8 & 5 & 3 & 3 & $\mathbf{3}$ & 0 & 0 & $\mathbf{0}$ \\
$\mathbf{3}$ & 10,20 & - & - & 5 & 8 & 6 & 8 & 10 & 8,4 & 1 & $\mathbf{2}$ & $\mathbf{1}$
\end{tabular}

Versuch, den 13. Juli 1898.

$$
\begin{array}{l|l|l|l|l|l|l|l|l|l|l|l|l}
\mathbf{1} \\
\mathbf{2} \\
\mathbf{3} & 9,30 & 30^{\circ} & 0 & 4 & 8 & 5 & 1 & 6 & \mathbf{2} & 0 & 0 & \mathbf{0} \\
& 9,34 & - & - & 5 & 5 & 5 & 1 & 3 & 2 & 0 & 0 & \mathbf{0} \\
\hline 9,37 & - & - & 7 & 10 & 9 & 1 & 6 & \mathbf{2} & \mathbf{2} & 5 & \mathbf{4}
\end{array}
$$


Die Erregbarkeit des undurchschnittenen Nerven ist, wie ja auch für andere Reize seit $\mathrm{Heidenhain} \mathrm{bekannt,} \mathrm{niedriger} \mathrm{als} \mathrm{die}$ des durchschnittenen. Im übrigen aber sind durchweg die oberen Stellen erregbarer als die unteren.

Um an möglichst unverletzten und durch die mechanische Reizung in keiner Weise in ibrer Erregbarkeit veränderten Nerven Versuche anzustellen, wurden Nerven nur immer ein einziges Mal, nämlich einmal oben, einmal nach 10-20 Sekunden in der Mitte und dann unten gereizt. Das Ergebniss war dasselbe. Es ergaben sich z. B. Reizerfolge wie: oben Zuckungshöhe 7,5, mitten 5,3, unten $0 \mathrm{~mm}$.

Hällstén hat dann weiter wie Heidenhain die Erregbarkeit des Nerven in der ganzen Länge seines Verlaufes gegenüber mechanischen Reizen untersuclst. Ich habe das nicht gethan, sondern mir genügte, wie gesagt, die Thatsache, dass der Nerv in seinen verschiedenen Stellen gegenüber den von mir angewendeten mechanischen Reizen sich verschieden verhielt und oben ausnahmslos eine grössere Erregbarkeit aufwies, als unten.

Worin lag nun aber wohl die Ursache in den verschiedenen Erfolgen der mechanischen Reizung bei Tigerstedt und bei mir, beziehungsweise Hällstén? Indem ich zunächst als selbstverständlich vorausschicke, dass ich die Tigerstedt'schen Angaben keinen Angenblick bezweifle, möchte ich bemerken, dass Tigers ted t meistens grössere und vor allen Dingen schmälere, gewissermaassen schärfere Gewichte angewendet hat, als ich.

Dies mag wohl neben deu allmählichen Aenderungen ier Erregbarkeit (siehe oben) die Hauptursache der verschiedenen Erfolge gewesen sein.

Herr Prof. Grützner hatte mir gleich von Anfang an die Aufgabe gestellt, die Wirkung verschiedener mechanischer Reize miteinander zu vergleichen, die zwar gleich grosse, aber der Art nach verschiedene Energiemengen in sich fassten.

Diese merkwürdigerweise noch kaum in Angriff genommene Frage konnte ich leider wegen Zeitmangel nicht so weit fördern, als ich es gern gethan hätte. Herr Prof. Grützner hat sie dann im Verein mit Herrn Dr. Bürker noch weiter fortgeführt und beabsichtigt, sie nach verschiedenen Richtungen hin noch eingebender 
zu bearbeiten. Das Wesentliche aller dieser Untersuchungen sei hier kurz mitgetheilt.

Wie gleich grosse, aber verschiedenartige Energiemengen auf Sinnesapparate wirken, ist meines Wissens noch wenig untersucht. Am leichtesten könnte man wohl derartige Versuche mit der Druckempfindlichkeit der $\mathrm{Haut}$ anstellen, indem man Gewichte von verschiedener Grösse und aus verschiedener Hohe auf die Haut auffallen lässt, und dafür sorgt, dass immer die Energiemengen gleich sind. Herr Prof. Grützner hatte früher gelegentlich einige Versuche derart angestellt, indem er die Gewichte auf einen Fingernagel fallen liess. Es ergab sich in Uebereinstimmung mit ähnlichen Untersuchungen von v. Frey ${ }^{1}$ ) und Anderen, dass schnelle Reize kräftiger wirken, als langsame.

Des weiteren hatte man akustische Reize untersucht. Um die Hörschärfe zu prüfen, musste man zunächst über verschiedenartige, aber subjectiv gleiche Reize verfügen. Diese glaubte man auf die Weise erzengen zu können, dass man eine Platte mit gleichen Energiemengen erschütterte. Man liess also beispielsweise auf dieselbe ein Gewicht von $p$ grm $h \mathrm{~cm}$ hoch und ein solches von $p_{1} \mathrm{grm} h_{1} \mathrm{~cm}$ hoch herabfallen und sorgte dafür, dass $p h=p_{1} h_{1}$ war, dass also z. B. das dreimal so schwere Gewicht nur ein Drittel so hoch herabfiel, wie das leichtere.

K. Vierordt ${ }^{2}$ ) aber konnte auf das Unzweifelhafteste zeigen, dass diese Annahmen nicht zutrafen; vielmehr ergab sich durchweg, dass das schwerere Gewicht einen viel stärkeren Schall erzeugte, als das leichtere. Ein Schrotkugelchen z. B. von $7 \mathrm{mg}$, welches $116,2 \mathrm{~mm}$ hoch auf eine Schiefertafel fiel, also eine Energie von $813 \mathrm{mg} \mathrm{mm}$ darstellte, erzeugte einen nahezu ebenso lauten Schall, wie ein anderes von $36,5 \mathrm{mg}$, bei einer Fallhöhe von $4,3 \mathrm{~mm}$, also einer Energie von nur $157 \mathrm{mg} \mathrm{mm}$. Genauere Messungen ergaben Vier ordt, dass gleiche Wirkungen etwa dann eintraten, wenn die Gleichung bestand, $p \sqrt{h}=p_{1} \sqrt{h_{1}}$.

Nahezu die gleiche Thatsache, aber so zu sagen in das Objective

1) M. v. Frey, Untersuchungen über die Sinnesfunctionen u. s. w., sächs. Gesellsch. der Wissenschaft Bd. 23, math.-physikal. Classe 1896 S. 175 (188).

2) K. Vierordt, Zeitschrift für Biologie Bd. 141878 S. 300. 
übersetzt, fand $O \mathrm{berbeck}^{1}$ ). Er konnte zeigen, dass von zwei verschieden schweren Kugeln, welche bei Entfaltung gleicher Energiemengen auf die Holzplatte eines Mikrophons fielen, die schwerere den Leitungswiderstand stärker herabsetzte, als die leichtere. Auch die Verhältnisse waren nahezu dieselben. Gleiche Wirkungen traten ein, wenn $p h^{0,622}=p_{1} h_{1}^{0,622}$ war.

Es fragte sich nun, wie verhalten sich die Nerven? Galt bei ihnen dasselbe Gesetz oder ein anderes?

Ehe wir jedoch diese Frage in Angriff nahmen, überzeugten wir uns nebenbei von der Richtigkeit der Vierordt'schen Angaben und prüften dieselben noch in folgenden Formen, zunächst an einer Marey'schen Trommel ${ }^{2}$ ), auf deren Aluminiumplatte verschiedene Gewichte aus verschiedenen Höhen herabfielen. Der Ausschlag des Zeichenhebels an der mit ihr in Verbindung stehenden Zeichentrommel wurde auf einem berussten Cylinder aufgezeichnet.

Die fallenden Gewichte waren Blei(Schrot)kugeln von verschiedener Grösse. Sie wurden zwischen den hohlen Blättern einer besonders dazu gebauten Pincette gehalten, die sich durch Druck von einander entfernten und die Kugel von bestimmter Höhe fallen liessen.

Aus vielen derartigen Versuchen sei einer herausgegriffen, bei welchem die Ausschläge des Zeichenhebels genau gleich gross waren, die Aufnahmeplatte der ersten Marey'schen Trommel also gleich stark erschüttert worden war. Dies trat ein, wenn herabfielen

$200 \mathrm{mg} 490 \mathrm{~mm}$, also bei einer Energie von $98000 \mathrm{mg} \mathrm{mm}$,

\begin{tabular}{|c|c|c|c|c|c|c|c|}
\hline 300 & n 200 & $n$ & $n$ & $"$ & $"$ & $"$ & $" 60000$ \\
\hline 441 & 80 & " & $n$ & $n$ & $n$ & $n$ & 35280 \\
\hline 300 & 40 & $"$ & $"$ & $n$ & $"$ & $"$ & 24000 \\
\hline 00 & $" \quad 20$ & $"$ & $\eta$ & n & $\eta$ & , & 16000 \\
\hline
\end{tabular}

Man sieht also, welch' ausserordentliches Uebergewicht die schwereren Gewichte über die leichteren hatten. Bei den schweren genügte eine Energie von $16000 \mathrm{mg} \mathrm{mm}$, um dieselbe Wirkung hervorzubringen, wie bei den leichten eine Energie von $98600 \mathrm{mg} \mathrm{mm}$, also etwa eine 6 mal so grosse.

1) A. Oberbeck, Wiedemann's Annalen. Bd. 23 S. 222. 1881. Dieses Gesetz ist sicher nicht allgemein gültig. Vgl. P. Starke, Wundt's philosoph. Studien Bd. 3 und 5. Grützner.

2) Es war dies die grössere Form der von Universitäts-Mechanikus E. Albrecht hierselbst nach Angabe von Hürthle gelieferten Trommel. 
Es wurden dann weiter Versuche mit der Platte eines Telephons angestellt, auf welche ebenfalls Schrotkugeln verschiedenen Gewichtes herabfielen. Damit die Kugeln gut liegen blieben und nicht $\mathrm{zu}$ wiederbolten Malen, wenn auch noch so schwach, in die Höhe hüpften oder gar herabrollten, war auf der genau horizontal gestellten Telephonplatte ein Stück feuchtes Fliesspapier aufgelegt und ihr Rand mit Papier, wie ein Schachteldeckel, umklebt.

Zunächst prüfte man die Erfolge mit dem Gehör an einem zweiten Telephon, welches durch ein grosses Zimmer von dem ersten getrennt war. Ohne weiteres konnte man (es betheiligten sich mehrere Personen an den Hörprüfungen) einen sicheren Unterschied zwischen der Schallstärke von grösseren und kleineren fallenden, gleiche Energiemengen repräsentirenden Gewichten (von $0,7,0,4$ und $0,2 \mathrm{~g}$ 2-8 $\mathrm{cm}$ Fallböhe) nicht mit Sicherheit wahrnehmen. Es wurden nahezu eben so viel richtige, wie falsche Antworten gegeben.

Wenn man aber in den Telephonkreis einen Siemens'schen Rheostat als Nebenschluss einschaltete und durch Stöpselung die Geräusche im Telephon immer schwächer werden liess, so verschwand das von dem grösseren Gewichte herrührende Geräusch bei einem kleineren Widerstand im Nebenschluss, als das von dem kleineren Gewicht herrührende. Derartige Untersuchungen sind, wie Jeder weiss, der hierüber einige Erfahrung hat, recht schwierig und umständlich. Ihr Ergebniss war aber doch ziemlich eindeutig. Es braucht kaum bemerkt zu werden, dass der Hörende natürlich nie wusste, um was es sich im einzelnen Falle handelte. Er hatte nur anzugeben und zu registriren, ob er ein bestimmtes Geräusch leiser oder lauter als ein anderes gehört hatte.

Waren diese Untersuchungen schwierig und umständlich und weil mit dem subjectiven Moment behaftet, immer gewissen Finwänden zugänglich, so war das Entgegengesetzte der Fall, sobald man die in dem Telephon erzeugten Ströme zur Reizung des Nerven benutzte. Nahezu ausnahmslos konnte hier gezeigt werden, dass das sehwerere Gewicht viel stärker reizte, als das leichtere, was ja auch von vornherein zu erwarten war.

Statt der vielen hierüber angestellten Versuche ein paar Versuchsbeispiele.

Versuch, den 25. Janụar 1899.

Das oben beschriebene Hämmerchen fällt auf die Mitte einer Platte eines älteren kleinen Telephons. Das Hämmerchen horizontal gestellt, drückt mit 
$0,12 \mathrm{~g}$ auf eine Wagschaale. Es werden ihm Gewichte von 0,13 und $0,38 \mathrm{~g}$ aus Messing aufgesetzt, sodass $0,25 \mathrm{~g}(p)$, beziehungsweise $0,5 \mathrm{~g}(P)$ auf die Platte fallen. Das Hämmerchen wird mit dem leichten Gewicht fast $21^{\circ}$, mit dem schweren $10^{\circ}$ hoch gehoben. Die Energiemengen bei dem Fall sind also nahezu gleich, keinesfalls ist die durch das schwerere Gewicht erzeugte grösser.

Das leichte Hämmerchen fällt 5 mal hintereinander in kurzen Pausen auf die Telephonplatte und es werden die ersten 5 Zuckungen $1-5$ (siehe Fig. 3) gezeichnet. Nach einer längeren Pause erfolgen noch 2 gleichartige Zuckungen 6 und 7. Dann fällt das grosse Gewicht 9 mal hintereinander und zeichnet die Zuckungen 8-16.

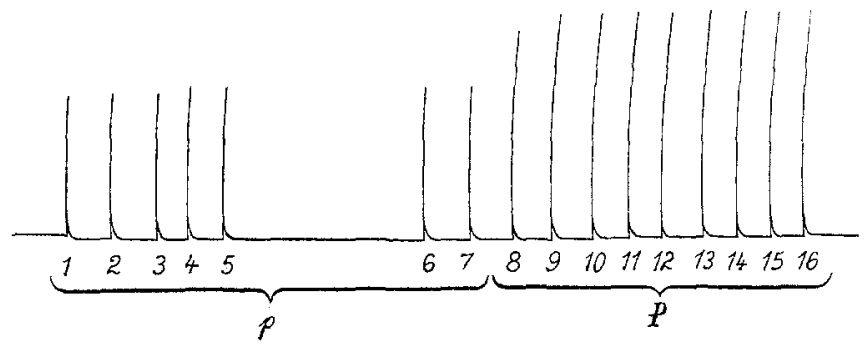

Fig. 3.

Versuch, den 29. Juli 1898.

Auf die Platte eines Siemens'schen Telephons fallen Schrotkugeln von verschiedenem Gewicht und verschiedener Höhe. Siemens'scher Rheostat im Nebenschluss. Galvanisches Präparat. Es wird festgestellt, bei welchem Widerstand die ersten Zuckungen erfolgen. Es fallen $282 \mathrm{mg} 150 \mathrm{~mm}$, beziehungsweise $141 \mathrm{mg} 300 \mathrm{~mm}$. Im ersten Fall traten bei $150 \mathrm{~S}$. Widerstand die gleichen Zuckungen ein, wie im zweiten bei $180 \mathrm{~S}$. Die ersten Zuckungen erschienen bei dem schweren Gewicht bei $60 \mathrm{~S}$., im zweiten bei $80 \mathrm{~S}$. Widerstand im Nebenschluss. Fallen die Gewichte 100 und $200 \mathrm{~mm}$ herab, so sind die entsprechenden Zahlen 145 und 200.

Versuch, den 7. Juni 1899.

Auf das im ersten Versuch gebrauchte Telephon, dessen Platte mit ein wenig feuchtem Fliesspapier belegt ist, fallen Schrotkugeln von 400 und $200 \mathrm{mg}$ 40 bezw. $80 \mathrm{~mm}$ hoch auf. Rheostat im Nebenschluss. Unpolarisirbare Elektroden in der Mitte des Nerven. Muskel am Grützner'schen Federmyographion, Spannung $=12 \mathrm{~g}$.

Beifolgende Figur 4. erläutert die Gesetzlichkeiten. Bei starken Strömen (Nebenschluss 1500 und 1000 S.) sind die Zuckungen, durch den Fall des grossen (P) und des kleinen Gewichtes (p) erzeugt, gleich gross. Bei $500 \mathrm{~S}$. Nebenschluss sind die ersten Zuckungen, die immer durch das grosse Gewicht erzeugt werden, noch maximal, das kleine erzeugt die beiden ersten Male keine Zuckung (o), nur das dritte Mal eine kleine Zuckung. Bei 600 S. Nebenschluss 
sind die Verhältnisse noch deutlicher. Die ersten hohen Zuckungen rühren von dem schwereren Gewicht, die ihnen folgenden von dem leichten her, dann folgen 4 Zuckungen hintereinander mit dem grossen, 4 hintereinander mit dem kleinen Gewicht and noch weiter verschiedene Gruppen von Zuckungen, von denen die kleinen stets von dem kleinen, die grossen von dem grossen Gewicht herrühren.

Die geringere Geschwindigkeit des grösseren Gewichtes bewegte also die Telephonplatte als Ganzes stärker, als die grössere Geschwindigkeit des kleineren Gewichtes. Im letzteren Falle mussten also mehr örtliche Erschütterungen, vielleicht auch mehr Wärme gebildet werden. Offenbar finden ganz ähnliche Vorgänge, worauf mich Herr Prof. Grützner aufmerksam machte, auch bei der Ansprache des Telephons mit verschiedenen Vocalen statt. Dem bekanntlich sehr schwach wirksamen $I$ entsprechen die leichteren Gewichte, dem viel stärker wirksamen $O$ oder $U$ die schwereren Gewichte.

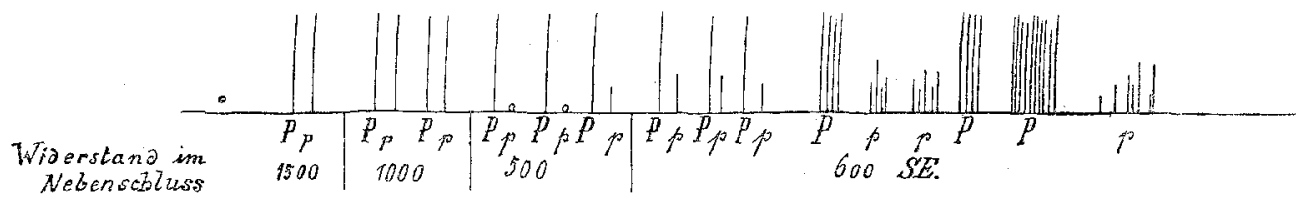

Fig. 4.

Ich wende mich nun nach diesen Abschweifungen zu unserer eigentlichen Frage, nämlich der unmittelbaren mechanischen Reizung des Nerven mit sehweren und leichten Gewichten.

Wenn man möglichst unverletzte, frische Nerven von kräftigen Fröschen untersucht, so findet man (umgekehrt wie bei dem Fall auf das Telephon) als Regel, dass das leichtere Gewicht stärker erregend wirkt, als das schwerere. Freilich wird man auch auf mannigfache Ausnahmen stossen, die sich aber immer erst nach längerer Versuchsdauer und bei mehr oder weniger geschädigten Nerven einstellen.

Wichtig ist bei all' diesen Versuchen, die nöthige Zeit zwischen den einzelnen mechanischen Reizen verstreichen zu lassen, worauf auch Tigerstedt mit Recht aufmerksam gemacht hat. Thut man dies nicht, so wird man von einer Fülle einander widersprechender Thatsachen geradezu erdrückt. Es ist das begreiflich, da, wie schon Tigerstedt ${ }^{1}$ ) gefunden hat, schwächere mechanische Reize die ört-

1) R. Tigerstedt, Mechanische Nervenreizung S. 44.

E. Pflüger, Archiv für Physiologie. Bd. 77 . 
liche Erregbarkeit erhöhen, stärkere oder oft wiederholte schwächere dagegen sie herabsetzen. Macht man da also nicht die nöthigen Erholungspausen, so erhält man die verschiedenartigsten Versuchsergebnisse.

\section{Folgende Versuche dienen zur Erläuterung.}

Versuch, den 29. Juli 1898.

Grosser kräftiger männlicher Grasfrosch, kurz vor $11 \mathrm{Uhr}$ getödtet. Ein Hüftnerv bis zum Kniegelenk frei präparirt, so dass er mit dem ganzen Unterschenkel in Verbindung bleibt; dann oben am Austritt unterbunden und abgeschnitten. Auf Platinelektroden gelegt, die mit einem Siemens'schen Telephon verbunden sind; auf seine Platte fallen die Schrotkugeln.

\begin{tabular}{|c|c|c|c|c|c|}
\hline Zeit & $\begin{array}{l}\text { Gewicht } \\
\text { in } \mathrm{mg}\end{array}$ & $\begin{array}{l}\text { Fallhöhe } \\
\text { in mm }\end{array}$ & $\begin{array}{l}\text { Energie in } \\
\mathrm{mg} \mathrm{mm}\end{array}$ & Wirkung & Bemerkungen \\
\hline 11,00 & 155 & 20 & $\begin{array}{c}3100 \\
-\end{array}$ & $\begin{array}{l}\text { Starke Zuckung } \\
\text { Keine Zackung }\end{array}$ & $\begin{array}{l}\text { Bei Reizung oben. } \\
\text {... mitten u. unten }\end{array}$ \\
\hline 一 & I & $\begin{array}{r}10 \\
5 \\
4\end{array}$ & $\begin{array}{r}1550 \\
775 \\
620\end{array}$ & $\begin{array}{l}\text { Zuckung } \\
\text { Schwache Zuckung } \\
\text { Ebenso }\end{array}$ & $\begin{array}{l}\text { Elektroden oben } \\
\text { angelegt } \\
\text { Jede Reizung }\end{array}$ \\
\hline 11,30 & $\begin{array}{l}441 \\
= \\
-\end{array}$ & $\begin{array}{l}10 \\
5 \\
3,5 \\
2 \\
1\end{array}$ & $\begin{array}{c}4410 \\
2205 \\
1323,5 \\
892 \\
441\end{array}$ & $\begin{array}{l}\text { Starke Zuckung } \\
\text { Ebenso } \\
\text { Zuckung } \\
\text { Ebenso } \\
\text { Zuckungen, aber } \\
\quad \text { nicht regelmässig }\end{array}$ & $\begin{array}{l}\text { mehrere Male } \\
\text { hinter einander } \\
\text { wiederholt }\end{array}$ \\
\hline 11,45 & $\underline{50}$ & $\begin{array}{c}10-100 \\
200 \\
150\end{array}$ & $\begin{array}{c}500-5000 \\
10000 \\
7500\end{array}$ & $\begin{array}{l}\text { Keine Zuckung } \\
\text { Zuckung } \\
\text { Schwache Zuckung }\end{array}$ & \\
\hline
\end{tabular}

Auf den Nerv und zwar auf sein oberes Ende wird ein Deckgläschen gelegt, auf welches die Gewichte herabfallen.

\begin{tabular}{|c|c|c|c|c|c|}
\hline Zeit & $\begin{array}{c}\text { Gewicht } \\
\text { in } \mathrm{mg}\end{array}$ & $\begin{array}{l}\text { Fallhöhe } \\
\text { in } \mathrm{mm}\end{array}$ & $\begin{array}{c}\text { Energie } \\
\text { in } \mathrm{mg} \mathrm{mm}\end{array}$ & Wirkung & $\begin{array}{c}\text { Be- } \\
\text { mer- } \\
\text { kungen }\end{array}$ \\
\hline $\begin{array}{l}11,51 \\
12,01\end{array}$ & $\stackrel{50}{-}$ & $\begin{array}{r}100 \\
50\end{array}$ & $\begin{array}{l}5000 \\
2500\end{array}$ & $\begin{array}{l}\text { Zuckung } \\
\text { Keine Zuckung }\end{array}$ & \\
\hline 12,02 & $\frac{155}{-}$ & $\begin{array}{l}20 \\
40 \\
60\end{array}$ & $\begin{array}{l}3100 \\
6200 \\
9300\end{array}$ & $\begin{array}{c}\text { Keine Zuckung } \\
\text { Ebenso } \\
\text { Zuckung }\end{array}$ & \\
\hline 12,10 & $\frac{441}{=}$ & $\begin{array}{l}30 \\
20 \\
15 \\
10\end{array}$ & $\begin{array}{r}13230 \\
8820 \\
6615 \\
4410\end{array}$ & $\begin{array}{c}\text { Zuckung } \\
\text { Schwache Zuckung } \\
\text { Ebenso } \\
\text { Ganz schw. Zuck. (selten) }\end{array}$ & \\
\hline
\end{tabular}


Bei den beiden Versuchen mögen etwa gleich stark gereizt haben die fettgedruckten Energiemengen. In dem Telephonversuch reizte also etwa gleich stark bei einem Gewicht von

$441 \mathrm{mg}$ eine Energiemenge von $882 \mathrm{mg} \mathrm{mm}$,

155

$50 " n$

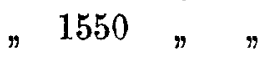

$" 10000$ "

In dem einfachen Fallversuch dagegen reizte etwa gleich stark bei einem Gewicht von

$441 \mathrm{mg}$ eine Energiemenge von $13230 \mathrm{mg} \mathrm{mm}$,

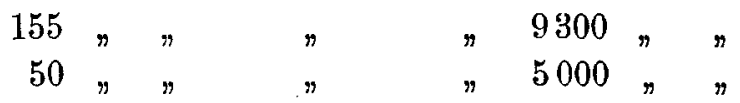

Ganz abgesehen von der Thatsache, dass hier die Energie der fallenden Gewichte viel sparsamer als elektrische Energie zur Ver wendung gebracht werden kann, während sie unmittelbar - aller-

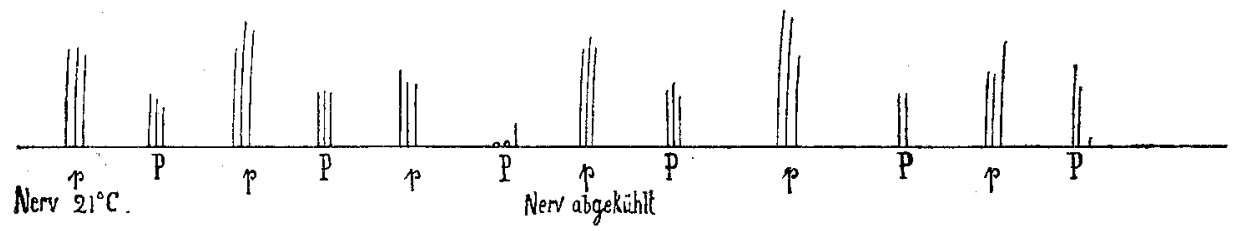

Fig. 5.

dings durch das Gläschen gemindert - den Nerven günstigsten Falls erst in 6 facher Stärke reizt, erkennt man, dass die kleineren Gewichte durch ihren Fall auf den Nerven diesen stärker reizen, als die grösseren.

Noch ein zweiter Versuch von den ausserordentlich vielen, welche mit dem Hämmerchen angestellt wurden, sei hier mitgetheilt.

$$
\text { Versuch, den 18. Februar } 1899 .
$$

Der Nerv lag in oben beschriebener Weise auf einem hohlen, rechteckigen Messingkästchen, auf feuchtem mit physiologischer Kochsalzlösung getränktem Fliesspapier. Das Hämmerchen $250 \mathrm{mg}(p)$ und $1000 \mathrm{mg}(P)$ schwer, fiel 40 bezw. $10 \mathrm{~mm}$. Durch das Kästchen floss Wasser von $21^{\circ} \mathrm{C}$. Der Nerv wurde stets an derselben! Stelle nahe seiner Mitte immer dreimal hinter einander in Pausen von etwa 10 Secunden gereizt, dann das Gewicht und die Fallhöhe verändert, und nach einer Pause von mindestens einer halben Minute wieder 3 Reizungen hintereinander ausgeführt.

Um Raum zu sparen, sind die Zuckungen etwas näher an einander gezeichnet, als sie es im Original waren (siehe Fig. 5). 
Man sieht, die mit $P$ bezeichneten, also durch den Fall des grossen Gewichtes erzeugten Zuckungen sind durchweg kleiner, als die mit $p$ bezeichneten. Ganz ähnliche Ergebnisse erhielt man auch, wenn die fallenden Gewichte 500 und $250 \mathrm{mg}$ betrugen (s. S. 192).

Mit wenigen Worten sei zum Schluss noch auf Versuche hingewiesen, in. denen der Nerv abgekühlt, beziehungsweise erwärmt und zugleich mechanisch gereizt wurde.

Dass Veränderung der Temperatur die physiologischen Vorgänge im Nerven in hohem Maasse beinflusst, insonderheit dass Wärme ihre Dauer verkürzt, Kälte sie verlängert, ist seit langer Zeit bekannt. Die gewöhnliche Angabe ging ferner dahin, dass die Erwärmung die Erregbarkeit erhöht, die Abkühlung sie dagegen herabsetzt. Diese Angabe ist aber für alle Fälle sicher nicht zutreffend, sondern es ist eher die entgegengesetzte richtig.

Richtig ist zwar im Allgemeinen die Thatsache, dass ein kaum wirksamer Reiz stark wirksam wird, wenn man den Nerven entweder im Ganzen oder diesseits des Reizes erwärmt, so dass der Reiz die erwärmte Stelle zu passieren hat. Richtig ist ferner, dass gleichartige Abkühlung die entgegengesetzte Wirkung ausübt und einen vorher wirksamen Reiz unwirksam macht.

Ganz anders aber fallen die meisten derartigen Versuche aus, wenn man den Reiz jenseits der erwärmten oder abgekühlten Stelle anbringt, so dass der Reiz gerade noeh in den Bereich der erwärmten oder abgekühlten Stelle zu liegen kommt, dann aber nur den völlig unveränderten Nerv zu durchsetzen hat.

In einer lehrreichen Arbeit haben Gotch und Macdonald ${ }^{1}$ ) gezeigt, dass dann für die meisten Reize ${ }^{2}$ ) die örtliche Erregbarkeit.

1) F. Gotch und J. S. Macdonald, Journal of physiol vol. 20 p. 247, 1896 und p. 255 .

2) Dass dies nicht für alle Reize gilt, dass z. B. der erwärmte Nerv für sehr schnell ablaufende elektrische Reize (Oeffnurgsinductionsströme u. a.) besser erregbar ist, als für langsame, auf die wieder der abgekühlte besser reagirt, wurde ebenfalls von Gotch und Macdonald in ihrer sorgfältigen und wichtigen Arbeit gezeigt, war aber - was die Forscher offenbar nicht wussten - schon v. Kries (Berichte der naturforsch. Gesellschaft in Freiburg Bd. 8 S. 2) festgestellt worden. Auch ich hatte denselben Schluss aus den Versuchen von Efron (Pflüger's Archiv Bd. 36 S. 488, 1886) gezogen, gebe aber gern zu, dass wie Gotch und Macdonald behaupten, unsere Versuche nicht eindeutig waren. Da es mir aber bei den Efron'schen Versuchen wesentlich daranf ankam, die verschiedene Erregbarkeit oberer und unterer Nervenstrecken festzustellen, so treffen die unserer Methode gemachten Ausstellngen nur zum kleinsten Theil zu. Grützner. 
[Reizbarkeit (Gad, J. Munk und Schultz), Aufnahmsfähigkeit (Schiff u. A.), excitability (Gotch und Macdonald)] bedeutend gesteigert, durch Wärme dagegen herabgesetzt wird. Die Leitungsfähigkeit (conductivity) freilich wird durch Wärme erhöht, durch Kälte herabgesetzt. Desshalb häufig die entgegengesetzten Erfolge, wenn der Reiz die erwärmte oder abgekühlte Strecke zu durchsetzen hat. Diese Erfolge sind dann aber nicht rein, sondern durch Veränderung der Leitungsfäbigkeit complicirt.

Es ist, wie mir Herr Prof. Grützner mittheilte, leicht, sich von der Richtigkeit der Angaben von Gotch und Macdonald zu überzeugen. Er bediente sich hierzu des oben erwähnten hohlen Messingkästchens; auf welchem der Nerv seiner ganzen Länge nach auf feuchtem Fliesspapier lag. Fs war eine Einrichtung getroffen, dass entweder 1. der ganze Nerv abgekühịt oder erwärmt, oder dass 2. die obere Hälfte allein, oder dass 3. die untere Hälfte allein erwärmt, beziehungsweise abgekühlt werden konnte. Im ersten Falle floss durch beide Kammern des Kästchens Wasser von derselben Temperatur hindurch, im zweiten nur dureh eine von beiden das temperirte Wasser, während Wasser mittlerer Temperatur stets dureh die andere Kammer hindurehfloss.

Folgende Versuche seien in Zahlen mitgetheilt.

Versuch, den 22. Juni 1899.

Grosser männlicher Grasfrosch, getödtet $10^{\mathrm{h}} 55^{\prime}$. Zimmertemperatur $20^{\circ} \mathrm{C}$. Nerv auf dem mit Wasser durchspülten Messingkästchen. Hammergewicht $500(P)$ und $250 \mathrm{mg}(p)$. Hubhöhen 20 and $40 \mathrm{~mm}$. Zwei Schläge folgten unmittelbar hintereinander; dann stets eine Pause von mindestens einer halben Minute.

\begin{tabular}{|c|c|c|c|c|}
\hline \multirow[t]{2}{*}{ Zeit } & \multicolumn{2}{|c|}{$\begin{array}{c}\text { Höhe der Zuckungen } \\
\text { in mm }\end{array}$} & & \multirow[t]{2}{*}{ Bemerkungen } \\
\hline & bei $p$ & bei $P$ & & \\
\hline $\begin{array}{c}\mathrm{h} \\
1000\end{array}$ & $\begin{array}{l}0 \\
0 \\
9 \\
9,5 \\
10 \\
8 \\
= \\
= \\
= \\
=\end{array}$ & $\begin{array}{l}- \\
\overline{-} \\
\overline{-} \\
\overline{0} \\
0 \\
0 \\
0 \\
10 \\
9\end{array}$ & $\begin{array}{l}\text { unten gereizt } \\
\text { mitten " } \\
\text { oben " } \\
\text { unten " } \\
\text { mitten } \\
\text { oben }\end{array}$ & $\begin{array}{l}\text { Wasser von } 17^{\circ} \mathrm{C} \text {. fliesst durch } \\
\text { das ganze Kästchen }\end{array}$ \\
\hline
\end{tabular}


(Fortsetzung.)

\begin{tabular}{|c|c|c|c|c|}
\hline \multirow[t]{2}{*}{ Zeit } & \multicolumn{2}{|c|}{$\begin{array}{l}\text { Höhe der Zuckungen } \\
\text { in mm }\end{array}$} & & \multirow[t]{2}{*}{ Bemerkungen } \\
\hline & bei $p$ & bei $P$ & & \\
\hline $\begin{array}{c}\text { h } \\
1005\end{array}$ & $\begin{array}{l}\overline{-} \\
\overline{8} \\
8,2 \\
\overline{8} \\
8,3\end{array}$ & $\begin{array}{l}6 \\
6 \\
\frac{7}{7} \\
\frac{7}{-} \\
-\end{array}$ & $\begin{array}{c}\text { mitten gereizt } \\
= \\
= \\
= \\
=\end{array}$ & Genau die gleiche Stelle \\
\hline 1010 & $\begin{array}{l}9 \\
9,7 \\
\overline{-} \\
10,5 \\
11,1 \\
-\end{array}$ & $\begin{array}{l}- \\
\overline{9,8} \\
9,7 \\
\overline{-} \\
\overline{13} \\
11,2\end{array}$ & $\begin{array}{l}= \\
= \\
= \\
=\end{array}$ & $\begin{array}{l}\text { Wasser von } 7^{\circ} \mathrm{C} \text {. durch die } \\
\text { obere Hälfte des Kästchens, } \\
\text { Wasser von } 17^{\circ} \mathrm{C} \text {. durch die } \\
\text { untere Hälfte des Kästchens }\end{array}$ \\
\hline 1015 & $\begin{array}{l}\overline{-} \\
\overline{9,5} \\
\overline{9} \\
\overline{10} \\
10\end{array}$ & $\begin{array}{l}8,4 \\
9,1 \\
\overline{-} \\
10 \\
9 \\
-\end{array}$ & $\begin{array}{l}- \\
= \\
z \\
z\end{array}$ & $\begin{array}{l}\text { Wasser von } 17^{\circ} \mathrm{C} \text {. oben und } \\
\text { unten }\end{array}$ \\
\hline
\end{tabular}

Der Versuch wurde in ähnlicher Art auch mit zweimaliger Anwendung von Wasser von $30^{\circ}$, welches die Erregbarkeit bedeutend herabsetzte, bis um $11^{\mathrm{h}} 15^{\prime}$. fortgesetzt. Sowohl wäbrend, als namentlich unmittelbar nach der Wärmewirkung ergab sich bei Wasser von $18^{\circ} \mathrm{C}$. oben und unten.

1115

$$
\begin{array}{|c|c|c|}
- & 1 & - \\
6 & 0,8 & - \\
8 & - & - \\
- & 3 & - \\
\overline{7,6} & 6 & - \\
4,5 & - & -
\end{array}
$$

Aus diesen und aus anderen ähnlichen Versuchen ergab sich einmal die Erregbarkeitssteigerung für mechanische Reize durch die Kälte und die Herabsetzung durch die Wärme. Es zeigte sich aber ferner vielfach, dass Kälte wesentlich die Erregbarkeit für die Reizung mit grösseren Gewichten erhöht (s. Fig. 5, in der $P$ bei mittlerer Temperatur sehr schwach, nach Ablüblung des Nerven aber 
stark wirksam war), Wärme dagegen die Erregung durch kleine. Gewichte noch zu Stande kommen lässt, während die grossen fast gar nicht mehr reizen. Letztere Versuche, die Herr Prof. Grützner allerdings noch nicht als abgeschlossen ansieht, sind desshalb nicht leicht anzustellen, weil eine irgend wie wirksame Wärme namentlich im Verein mit mechanischen Reizen die Erregbarkeit eben überhaupt so schnell herabsetzt, dass nur ein fortwährender Wechsel zwischen grossen und kleinen Gewichten Schlüsse gestattet. Grosse Gewichte schädigen einen erwärmten Nerven ausserordentlich schnell, dem abgekühlten dagegen machen sie verhältnissmässig wenig, ja sie sind da im Gegentheil als Reize eher wirksamer, als die kleinen Gewichte.

Sollte sich, woran kaum zu zweifeln ist, dieses Gesetz auch in weiteren Grenzen bestätigen, so würde es eine interessante Ergänzung zu der verschiedenen Erregbarkeit abgekühlter und erwärmter Nerven gegenüber schnellen und langsamen elektrischen. Reizen bilden.

\section{Zusammenfassende Schlussbetrachtungen.}

Fasse ich hiernach die wesentlichen Ergebnisse obiger Arbeit zusammen, so ergab sich für den Hüftnerv des Frosches, dass seine Erregbarkeit oben und unten für jähe elektrische Reize ziemlich gleich, für langsam ansteigende dagegen unten viel kleiner, als oben war. Die Stromstärken mussten hier etwa auf das Doppelte. erhöht werden, um unten eben so stark zu wirken, wie oben.

Chemische Reize wirkten dagegen unzweifelhaft unten viel stärker, als oben. Ja oben angebrachte schwache Reize wirkten geradezu hemmend und verlängerten den Muskel. Andauernde, einigermaasen gleichmässige Tetani erzeugten sie nicht oder selten, und erst nach sehr langer Einwirkung.

Mechanische Reize (Fallende Gewichte) wirkten oben stets besser, als unten. Wendete man gleiche Energiemengen an, indem man die kleineren Gewichte von entsprechend grösseren Höhen herabfallen liess, als die grösseren, so wirkten in der Regel die kleineren mit grösserer Geschwindigkeit auf den Nerven aufschlagenden Gewichte besser, als die grossen mit geringerer Geschwindigkeit fallenden. Es bestände hiernach eine Aehnlichkeit mit dem elektrischen Erregungsgesetz von Du Bois-Reymond, welches, wenn 
auch nicht allgemein, so doch für den Hüftnerven des Frosches gültig ist.

Was kann man nun aus allen diesen Versuchen über das eigentliche Wesen der Nervenerregung, sowie dieselbe unter ganz normalen Bedingungen abläuft und von Ort zu Ort fortschreitet, für Schlüsse machen? Die Antwort ist sehr einfach. Streng genommen gar keine. Wenn beispielsweise ein langes Wasserrohr aus einem Behälter Wasser leitet, die Röhren selbst aber, wenn auch durchweg. von gleicher Lichtung, doch von sehr verschiedenem Material und verschiedener Wanddicke sind, so kann das Wasser in den verschiedenen Röhren in durchaus gleichartiger Weise mit gleichem Gefälle strömen. Wenn aber auf einem Röhrenstück ein. schwerer Stein lastet, so wird dasselbe, wenn dünnwandig, zusammengedrückt werden. Ist es in noch höherem Maasse nachgiebig, so kann eine andere Schädigung, z. B. eine starke Herabsetzung des Luftdruckes, dasselbe erweitern. So ähnlich hat man sich nach Herrn Prof. Grützner die Einwirkung verschiedener Reize auf den Nerven in seinem Verlauf vorzustellen. Der erste "Reiz", wenn man so sagen darf, hemmte den Wasserabfluss, der zweite beförderte ihn. An andern Stellen der Wasserleitungsrohre würden dieselben Schädigungen gar keinen Einfluss haben.

Man darf also, wie namentlich Gotch und Macdonald zuerst des Genaueren ausgeführt haben, nicht schlechtweg von Erregbarkeit eines Nerven oder von verschiedener Erregbarkeit eines und desselben Nerven an versehiedenen Stellen seines Verlaufes sprechen, sondern man muss stets von Erregbarkeit gegenüber bestimmten Reizen recien, und diese kann dann, wie wir oben gesehen haben, an denselben Stellen gegenüber verschiedenen Reizen sehr verschieden ausfallen. Aber ich betone noch einmal, einen Rückschluss auf den normalen Vorgang der Erregung gestatten alle diese Versuche ohne Weiteres nicht. Sie thun dies eben so wenig, als häufiger oder leichter zu erzeugende Störungen, welche vielleicht in dünnwandigen Wasserröhren eintreten, einen Schluss erlauben, dass unter normalen Verhältnissen auch in diesen dünnen Röhren das Wasser mit anderem Gefälle als in den dicken Röhren fliesst.

Es fragt sich aber weiter, ist diese Annahme wahrscheinlich? Sollte nicht doch, um bei obigem Beispiel zu bleiben, die Strömung des Wassers in den dünnwandigen Röhren unter normalen Verhältnissen eine andere sein, als in den dickwandigen? nun für diese 
Wahrscheinlichkeit babe ich mich schon oben ausgesprochen, da Reizleitung und Reizaufnahme, wenn auch verschiedene, doch nicht so weit verschiedene Vorgänge sein können ${ }^{1}$ ).

Ein langer, durchweg gleichartig gebauter Nerv, der auch keine Nebenäste abgiebt, wie der Phrenicus, wird sicher, wie ja auch direct gefunden wurde, viel einfachere, durchweg gleichartige Verhältnisse und Erregbarkeiten darbieten, als andere in ihrem anatomischen Bau verschiedene Nerven. Wie freilich die normalen Erregungswellen in den verschiedenen Nerven ablaufen, ist weder für den einen, noch für den andern bekannt. Ja für den Ablauf künstlicher, dem Nerven von aussen zugeführter Reize, herrschen sogar noch, wie oben mitgetheilt, die verschiedenartigsten Ansichten.

Und wenn nun gar die Nerven, wie man heut zu Tage mehr und mehr annimmt, nicht die einfachen Telegraphendrähte sind, in denen nur durchweg gleichartige, nur in ihrer Stärke verschiedene Vorgänge entstehen und ablaufen, sondern wie E. Hering ${ }^{2}$ ) kürzlich in geistreicher Weise entwickelt hat, „ein Bündel lebendiger Arme" sind, welche von den Elementarwesen des Nervensystems, das ist den Ganglienzellen, ausgestreckt werden, und so wie diese specifisch verschieden sind, auch ihrerseits qualitativ verschiedener Erregung fähig sind, so ist es klar, dass der Begriff der Erregbarkeit immer weiter und weiter werden muss und sich nicht bloss darauf beschränken darf, wie ein Nerv gegenüber einem Oeffnungsinductionsschlag reagirt.

1) Vgl. hierüber die eben erschienenen, wichtigen Untersuchungen von L. Hermann, Pflüger's Archiv Bd. 75 S. 574, 1899, sowie die früheren von J. L. Hoorweg, ebenda Bd. 53 S. 587, 1893.

2) E. Heřing, Zur Theorie der Nerventhätigkeit. Leipzig 1899. 